\title{
On the global well-posedness of a generalized 2D Boussinesq equations
}

\author{
Junxiong Jia, Jigen Peng and Kexue Li
}

Abstract. In this paper, we consider the global solutions to a generalized 2D Boussinesq equation

$$
\left\{\begin{array}{l}
\partial_{t} \omega+u \cdot \nabla \omega+\nu \Lambda^{\alpha} \omega=\theta_{x_{1}} \\
u=\nabla^{\perp} \psi=\left(-\partial_{x_{2}}, \partial_{x_{1}}\right) \psi, \quad \Delta \psi=\Lambda^{\sigma}(\log (I-\Delta))^{\gamma} \omega \\
\partial_{t} \theta+u \cdot \nabla \theta+\kappa \Lambda^{\beta} \theta=0 \\
\omega(x, 0)=\omega_{0}(x), \quad \theta(x, 0)=\theta_{0}(x),
\end{array}\right.
$$

with $\sigma \geq 0, \gamma \geq 0, \nu>0, \kappa>0, \alpha<1$ and $\beta<1$. When $\sigma=0, \gamma \geq 0$, $\alpha \in[0.95,1)$ and $\beta \in(1-\alpha, g(\alpha))$, where $g(\alpha)<1$ is an explicit function as a technical bound, we prove that the above equation has a global and unique solution in suitable functional space.

Mathematics Subject Classification. 76D03, 76D05.

Keywords. Generalized 2D Boussinesq equation, Global regularity,

Supercritical Boussinesq equations, Regularization effect.

\section{Introduction}

The aim of this paper is prove that the following generalized 2D Boussinesq equation has a global solution in suitable functional settings.

$$
\left\{\begin{array}{l}
\partial_{t} \omega+u \cdot \nabla \omega+\nu \Lambda^{\alpha} \omega=\theta_{x_{1}} \\
u=\nabla^{\perp} \psi=\left(-\partial_{x_{2}}, \partial_{x_{1}}\right) \psi, \quad \Delta \psi=\Lambda^{\sigma}(\log (I-\Delta))^{\gamma} \omega \\
\partial_{t} \theta+u \cdot \nabla \theta+\kappa \Lambda^{\beta} \theta=0 \\
\omega(x, 0)=\omega_{0}(x), \quad \theta(x, 0)=\theta_{0}(x)
\end{array}\right.
$$

where $\omega=\omega(x, t), \psi=\psi(x, t)$ and $\theta=\theta(x, t)$ are scalar functions of $x \in \mathbb{R}^{2}$ and $t \geq 0, u=u(x, t): \mathbb{R}^{2} \rightarrow \mathbb{R}^{2}$ is a vector field, $0<\alpha<1,0<\beta<1, \sigma \geq 0$ 
and $\gamma \geq 0$ are real parameters, and $\Lambda=(-\Delta)^{\frac{1}{2}}$ and $\Lambda^{\sigma}$ are Fourier multiplier operators with

$$
\widehat{\Lambda^{\sigma}} f(\xi)=|\xi|^{\sigma} \widehat{f}(\xi) .
$$

This generalized 2D Boussinesq equation proposed in [1] firstly. Chae and $\mathrm{Wu}[1]$ proved that the above vorticity equation does have the velocity formation as follows

$$
\left\{\begin{array}{l}
\partial_{t} v+u^{\perp}\left(\nabla^{\perp} \cdot v\right)+\nu \Lambda^{\alpha} v=-\nabla p+\theta e_{2}, \\
\nabla \cdot v=0, \quad u=\Lambda^{\sigma}(\log (I-\Delta))^{\gamma} v, \\
\partial_{t} \theta+u \cdot \nabla \theta+\kappa \Lambda^{\beta} \theta=0, \\
v(x, 0)=v_{0}(x), \quad \theta(x, t)=\theta_{0}(x) .
\end{array}\right.
$$

Obviously, the above model can be seen as a generalization of the 2D Boussinesq equations.

From physical view, Boussinesq type equations model the oceanic and atmospheric motions [2]. From the mathematical view, the fully viscos model with $\nu>0, \kappa>0, \alpha=\beta=2$ is the simplest one to study. And the most difficult one for the mathematical study is the inviscid model, that is when $\nu=\kappa=0$. In addition, the $2 \mathrm{D}$ Boussinesq equations acts very similar to the 3D Euler and Navier-Stokes equations, so it is hoped that the study of the 2D Boussinesq equations may shed light on the global regularity problem concerning the 3D Euler and Navier-Stokes equations.

Now, there are numerous studies about 2D Boussinesq equations. Chae [3] proved the global in time regularity for the 2D Boussinesq system with either the zero diffusivity or the zero viscosity. In 2010, further progress has been made by Hmidi et al. who proved the global regularity when the full Laplacian dissipation is replaced by the critical dissipation represented in terms of $\sqrt{-\Delta}[4,5]$. Recently, Miao and Xue [6] generalized the results to accommodate both fractional dissipation and fractional thermal diffusion. Some results about 3D case have been obtained in [8] by Hmidi et al. At the same time, some other generalized models have been considered. Lai et al. [9] studied the initial boundary value problem of 2D Boussinesq equations over a bounded domain with smooth boundary. Wang and Zhang [7] discussed the global wellposedness for the 2D Boussinesq system with the temperature-dependent viscosity and thermal diffusivity. Wu and Xue [10] showed that there is a global unique solution to the two-dimensional inviscid Bénard system with fractional diffusivity system.

We note that Chae and $\mathrm{Wu}$ [1] only studied system (1.1) in the case $\nu>0, \kappa=0, \alpha=0$. Concerning the other cases, can we get similar results as in 2D Boussinesq system for system (1.1) which is a meaningful generalization. In this paper, we focus on the case $\nu>0, \kappa>0,0<\alpha<1$ and $0<\beta<1$. Obviously, $\alpha$ and $\beta$ should satisfy the relation $\alpha+\beta \geq 1$, for the maximal gain of $\alpha$ derivative from the dissipation term should at least roughly compensate the loss of one derivative in $\theta$ in the vorticity equation of system (1.1) with the help of the diffusion effect in the temperature equation. For brevity, we always set $\nu=\kappa=1$ in the following. We shall adopt the subtle method used in 
$[1,4-6]$ to study the coupled effects of the generalized system. More precisely, we have the following result.

Theorem 1.1. Consider the generalized Boussinesq equations (1.1) with $\sigma=0$ and $\gamma \geq 0$. Let $\alpha \in\left[\frac{19}{20}, 1\right), \beta \in(1-\alpha, g(\alpha))$ with $g(\alpha):=\min \left\{2-2 \alpha, \frac{8}{3} \alpha-2\right.$, $\left.\frac{5 \alpha(1-\alpha)}{11-10 \alpha}\right\}$. Assume the initial data $\left(\omega_{0}, \theta_{0}\right)$ satisfies $\omega_{0} \in L^{2} \cap L^{p}$ for any $p>2$ and $\theta_{0} \in H^{1-\alpha} \cap B_{\infty, 1}^{1-\alpha+\epsilon}$ for arbitrary small $\epsilon>0$. Then (1.1) has a unique global solution $(\omega, \theta)$ satisfying for any $t>0$,

$$
\begin{aligned}
& \omega \in L_{t}^{\infty} L^{2} \cap L_{t}^{\infty} L^{p} \cap L_{t}^{1} B_{\infty, 1}^{0, \gamma} \\
& \theta \in L_{t}^{\infty}\left(H^{1-\alpha} \cap B_{\infty, 1}^{1-\alpha+\epsilon}\right) \cap L_{t}^{1}\left(H^{1-\alpha+\beta} \cap B_{\infty, 1}^{1-\alpha+\beta+\epsilon}\right) .
\end{aligned}
$$

For the definitions of Besov space $B_{p, q}^{s}$, generalized Besov space $B_{p, q}^{s, \gamma}$ and mixed space-time Besov space see the next section below. Now, we should give some comments.

Remark 1.2. We know that the case for $\alpha<1, \beta<1$ and $\alpha+\beta \geq 1$ is nontrivial. Until now there is no effective way to treat this case, for the regularization from the fractional diffusion term not strong enough. So we have to exploit the structure of the system to overcome the difficulty. In this paper, the method is workable but very restrictive.

Remark 1.3. In our theorem, we need $\beta$ smaller than a very complicated function. It is a technical assumption. In common sense, the term $\Lambda^{\beta}$ is a good term when $\beta$ is large. So we can gauss that the result in Theorem 1.1 is hold for $\alpha \in\left[\frac{19}{20}, 1\right), \beta \in(1-\alpha, 1)$.

To prove Theorem 1.1, there are two main difficulties. Firstly, following the procedure as in [1], we will encounter the operator like $\mathcal{R}_{\alpha}=\Lambda^{-\alpha} \partial_{1}$ which is different from Riesz Transform and is not a bounded operator in $L^{p}$ space. So the technique used in [1] can not be used here without significant changes. On the other hand, considering the structure of the system (1.2), we hardily obtain the $L^{2}$ estimates of $v$. Hence, the techniques used in [6] also need lots of nontrivial changes.

The paper is organized as follows. In Sect. 2, we list some useful results about Besov space and some estimates which will be used in our proof. Section 3 is devoted to prove some priori estimates which are the main part of this paper. In Sect. 4, we give the proof of the uniqueness part of Theorem 1.1. Finally, some technical lemmas are shown in Sect. 5 .

\section{Preliminaries}

Throughout this paper we will use the following notations.

- For any tempered distribution $u$ both $\widehat{u}$ and $\mathcal{F} u$ denote the Fourier transform of $u$.

- For every $p \in[1, \infty],\|\cdot\|_{L^{p}}$ denotes the norm in the Lebesgue space $L^{p}$. 
- The norm in the mixed space-time Lebesgue space $L^{p}\left([0, T] ; L^{r}\left(\mathbb{R}^{d}\right)\right)$ is denoted by $\|\cdot\|_{L_{T}^{p} L^{r}}$ (with the obvious generalization to $\|\cdot\|_{L_{T}^{p} X}$ for any normed space $\mathrm{X}$ ).

- For any pair of operators $P$ and $Q$ on some Banach space $X$, the commutator $[P, Q]$ is given by $P Q-Q P$.

Then, we give a short introduction to the Besov type space. Details about Besov type space can be found in [11] or [12]. There exist two radial positive functions $\chi \in \mathcal{D}\left(\mathbb{R}^{d}\right)$ and $\varphi \in \mathcal{D}\left(\mathbb{R}^{d} \backslash\{0\}\right)$ such that

- $\chi(\xi)+\sum_{q \geq 0} \varphi\left(2^{-q} \xi\right)=1 ; \forall q \geq 1, \operatorname{supp} \chi \cap \operatorname{supp} \varphi\left(2^{-q} \cdot\right)=\phi$,

- $\operatorname{supp} \varphi\left(2^{-j} \cdot\right) \cap \operatorname{supp} \varphi\left(2^{-k} \cdot\right)=\phi$, if $|j-k| \geq 2$,

For every $v \in S^{\prime}\left(\mathbb{R}^{d}\right)$ we set

$$
\Delta_{-1} v=\chi(D) v, \quad \forall q \in \mathbb{N}, \quad \Delta_{j} v=\varphi\left(2^{-q} D\right) v \quad \text { and } \quad S_{j}=\sum_{-1 \leq m \leq j-1} \Delta_{m} .
$$

The homogeneous operators are defined by

$$
\dot{\Delta}_{q} v=\varphi\left(2^{-q} D\right) v, \quad \dot{S}_{j}=\sum_{m \leq j-1} \dot{\Delta}_{j} v, \quad \forall q \in \mathbb{Z} .
$$

One can easily verifies that with our choice of $\varphi$,

$$
\begin{gathered}
\Delta_{j} \Delta_{k} f=0 \quad \text { if } \quad|j-k| \geq 2 \\
\Delta_{j}\left(S_{k-1} f \Delta_{k} f\right)=0 \quad \text { if }|j-k| \geq 5 .
\end{gathered}
$$

As in Bony's decomposition, we split the product $u v$ into three parts

$$
u v=T_{u} v+T_{v} u+R(u, v),
$$

with

$$
\begin{aligned}
& T_{u} v=\sum_{j} S_{j-1} u \Delta_{j} v, \\
& R(u, v)=\sum_{j} \Delta_{j} u \widetilde{\Delta}_{j} v
\end{aligned}
$$

where $\widetilde{\Delta}_{j}=\Delta_{j-1}+\Delta_{j}+\Delta_{j+1}$.

Let us now define inhomogeneous Besov spaces. For $(p, q) \in[1,+\infty]^{2}$ and $s \in \mathbb{R}$ we define the inhomogeneous Besov space $B_{p, q}^{s}$ as the set of tempered distributions $u$ such that

$$
\|u\|_{B_{p, q}^{s}}:=\left(2^{j s}\left\|\Delta_{j} u\right\|_{L^{p}}\right)_{\ell^{q}}<+\infty .
$$

The homogeneous Besov space $\dot{B}_{p, q}^{s}$ is defined as the set of $u \in S^{\prime}\left(\mathbb{R}^{d}\right)$ up to polynomials such that

$$
\|u\|_{\dot{B}_{p, q}^{s}}:=\left(2^{j s}\left\|\dot{\Delta}_{j} u\right\|_{L^{p}}\right)_{\ell^{q}}<+\infty .
$$

Notice that the usual Sobolev spaces $H^{s}$ coincide with $B_{2,2}^{s}$ for every $s \in \mathbb{R}$ and that the homogeneous spaces $\dot{H}^{s}$ coincide with $\dot{B}_{2,2}^{s}$. 
For $s \in(0,1)$ and $1 \leq p, q \leq \infty$, we can define Besov spaces equivalently as follows

$$
\begin{aligned}
\|u\|_{\dot{B}_{p, q}^{s}} & =\left(\int_{\mathbb{R}^{d}} \frac{\left(\|u(x+t)-u(x)\|_{L^{p}}\right)^{q}}{|t|^{d+s q}} d t\right)^{1 / q} \\
\|u\|_{B_{p, q}^{s}} & =\|u\|_{L^{p}}+\left(\int_{\mathbb{R}^{d}} \frac{\left(\|u(x+t)-u(x)\|_{L^{p}}\right)^{q}}{|t|^{d+s q}} d t\right)^{1 / q} .
\end{aligned}
$$

When $q=\infty$, the expressions are interpreted in the normal way.

We shall need some mixed space-time spaces. Let $T>0$ and $\rho \geq 1$, we denote by $L_{T}^{\rho} B_{p, q}^{s}$ the space of distribution $u$ such that

$$
\|u\|_{L_{T}^{\rho} \dot{B}_{p, q}^{s}}:=\left\|\left(2^{j s}\left\|\dot{\Delta}_{j} u\right\|_{L^{p}}\right)_{\ell^{q}}\right\|_{L_{T}^{\rho}}<+\infty .
$$

We say that $u$ belongs to the space $\widetilde{L}_{T}^{\rho} B_{p, q}^{s}$ if

$$
\|u\|_{\widetilde{L}_{T}^{\rho} \dot{B}_{p, q}^{s}}:=\left(2^{j s}\left\|\dot{\Delta}_{j} u\right\|_{L_{T}^{\rho} L^{p}}\right)_{\ell^{q}}<+\infty .
$$

Through a direct application of the Minkowski inequality, the following links between these spaces is true [13]. Let $\varepsilon>0$, then

$$
\begin{aligned}
& L_{T}^{\rho} B_{p, q}^{s} \hookrightarrow \widetilde{L}_{T}^{\rho} B_{p, q}^{s} \hookrightarrow L_{T}^{\rho} B_{p, q}^{s-\varepsilon}, \quad \text { if } q \geq \rho, \\
& L_{T}^{\rho} B_{p, q}^{s+\varepsilon} \hookrightarrow \widetilde{L}_{T}^{\rho} B_{p, q}^{s} \hookrightarrow L_{T}^{\rho} B_{p, q}^{s}, \quad \text { if } \rho \geq q .
\end{aligned}
$$

Then, we give the definition of a generalized Besov spaces which include an algebraic part of the modes. For $s, \gamma \in \mathbb{R}$ and $1 \leq p, q \leq \infty$, the generalized Besov spaces $\dot{B}_{p, q}^{s, \gamma}$ and $B_{p, q}^{s, \gamma}$ are defined by

$$
\begin{aligned}
\|u\|_{\dot{B}_{p, q}^{s, \gamma}} & :=\left\|2^{j s}(1+|j|)^{\gamma}\right\| \dot{\Delta}_{j} u\left\|_{L^{p}}\right\|_{\ell^{q}}<\infty, \\
\|u\|_{\dot{B}_{p, q}^{s, \gamma}} & :=\left\|2^{j s}(1+|j|)^{\gamma}\right\| \Delta_{j} u\left\|_{L^{p}}\right\|_{\ell^{q}}<\infty .
\end{aligned}
$$

The space $L_{t}^{\rho} B_{p, q}^{s, \gamma}, L_{t}^{\rho} \dot{B}_{p, q}^{s, \gamma}, \widetilde{L}_{t}^{\rho} B_{p, q}^{s, \gamma}$ and $\widetilde{L}_{t}^{\rho} \dot{B}_{p, q}^{s, \gamma}$ are defined similar as in Besov space.

Bernstein type inequalities for fractional derivatives and Osgood inequality are often used in our proof. For reader's convenience, we list them here.

Lemma 2.1. Let $\alpha \geq 0$. Let $1 \leq p \leq q \leq \infty$.

(1) If u satisfies

$$
\sup \hat{u} \subset\left\{\xi \in \mathbb{R}^{d}:|\xi| \leq K 2^{j}\right\},
$$

for some integer $j$ and a constant $K>0$, then

$$
\left\|(-\Delta)^{\alpha} u\right\|_{L^{q}\left(\mathbb{R}^{d}\right)} \leq C_{1} 2^{2 \alpha j+j d\left(\frac{1}{p}-\frac{1}{q}\right)}\|u\|_{L^{p}\left(\mathbb{R}^{d}\right)} .
$$

(2) If u satisfies

$$
\sup \widehat{u} \subset\left\{\xi \in \mathbb{R}^{d}: K_{1} 2^{j} \leq|\xi| \leq K_{2} 2^{j}\right\}
$$

for some integer $j$ and constants $0<K_{1} \leq K_{2}$, then

$$
C_{1} 2^{2 \alpha j}\|u\|_{L^{q}\left(\mathbb{R}^{d}\right)} \leq\left\|(-\Delta)^{\alpha} u\right\|_{L^{q}\left(\mathbb{R}^{d}\right)} \leq C_{2} 2^{2 \alpha j+j d\left(\frac{1}{p}-\frac{1}{q}\right)}\|u\|_{L^{p}\left(\mathbb{R}^{d}\right)}
$$

where $C_{1}$ and $C_{2}$ are constants depending on $\alpha, p$ and $q$ only. 
Lemma 2.2. Let $\alpha(t)>0$ be a locally integrable function. Assume $\omega(t) \geq 0$ satisfies

$$
\int_{0}^{\infty} \frac{1}{\omega(r)} d r=\infty
$$

Suppose that $\rho(t)>0$ satisfies

$$
\rho(t) \leq a+\int_{t_{0}}^{t} \alpha(s) \omega(\rho(s)) d s
$$

for some constant $a \geq 0$. Then if $a=0$, then $\rho=0$; if $a>0$, then

$$
-\Omega(\rho(t))+\Omega(a) \leq \int_{t_{0}}^{t} \alpha(\tau) d \tau,
$$

where

$$
\Omega(x)=\int_{x}^{1} \frac{1}{\omega(r)} d r .
$$

At the end of this section, we collect some useful estimates for the smooth solutions of the following linear transport-diffusion equation

$$
\left\{\begin{array}{l}
\partial_{t} \theta+u \cdot \nabla \theta+\Lambda^{\beta} \theta=f, \quad \beta \in[0,1] \\
\operatorname{div} u=0, \quad \theta(x, 0)=\theta_{0}(x) .
\end{array}\right.
$$

The following Lemmas can be found in $[6,14-16]$.

Lemma 2.3. Let $u$ be a smooth divergence-free vector field of $\mathbb{R}^{d}$ and $\theta$ be a smooth solution of Eq. (2.5). Then for every $p \in[1, \infty]$ we have

$$
\|\theta(t)\|_{L^{p}} \leq\left\|\theta_{0}\right\|_{L^{p}}+\int_{0}^{t}\|f(\tau)\|_{L^{p}} d \tau .
$$

Lemma 2.4. Let $u$ be a smooth divergence-free vector field of $\mathbb{R}^{d}$ with vorticity $\omega$ be a smooth solution of Eq. (2.5). Then for every $(p, \rho) \in(1, \infty) \times[1, \infty]$, we have

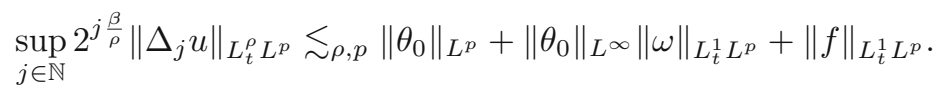

Lemma 2.5. Let $-1<s<1,\left(\rho, \rho_{1}, p, r\right) \in[1, \infty]^{4}, \rho_{1} \leq \rho$ and $u$ be a divergence-free vector field belonging to $L_{\text {loc }}^{1}\left(\mathbb{R}^{+} ; \operatorname{Lip}\left(\mathbb{R}^{d}\right)\right)$. We consider a smooth solution $\theta$ of the Eq. (2.5), then there exists $C>0$ such that for every $t \in \mathbb{R}^{+}$,

$$
\|\theta\|_{\widetilde{L}_{t}^{\infty} B_{p, q}^{s}}+\left\|\left(I d-\Delta_{-1}\right) \theta\right\|_{\widetilde{L}_{t}^{\rho} B_{p, q}^{s+\frac{\beta}{\rho}}} \leq C e^{C U(t)}\left(\left\|\theta_{0}\right\|_{B_{p, q}^{s}}+\|f\|_{L_{t}^{1} B_{p, q}^{s}}\right),
$$

and

$$
\|\theta\|_{\widetilde{L}_{t}^{\rho} \dot{B}_{p, q}^{s+\frac{\beta}{\rho}}} \leq C e^{C U(t)}\left(\left\|\theta_{0}\right\|_{\dot{B}_{p, q}^{s}}+\|f\|_{\widetilde{L}_{t}^{\rho_{1}} \dot{B}_{p, q}^{s+\frac{\beta}{\rho_{1}}}-\beta}\right),
$$

where $U(t):=\int_{0}^{t}\|\nabla u(\tau)\|_{L^{\infty}} d \tau$. 


\section{Commutator estimates}

First we recall a pseudo-differential operator $\mathcal{R}_{\alpha}$ defined by $\mathcal{R}_{\alpha}:=\Lambda^{-\alpha} \partial_{1}$, $0<\alpha<1$. Considering $\mathcal{R}_{\alpha}=\Lambda^{1-\alpha} \mathcal{R}$, where $\mathcal{R}$ is the usual Riesz transform, we call $\mathcal{R}_{\alpha}$ the modified Riesz transform. The following theorem can be found in $[6]$.

Theorem 3.1. Let $0<\alpha<1, q \in \mathbb{N}$ and $\mathcal{R}_{\alpha}:=\frac{\partial_{1}}{\Lambda^{\alpha}}$ be the modified Riesz transform.

(1) Let $\chi \in \mathcal{D}\left(\mathbb{R}^{d}\right)$. Then for every $(p, s) \in[1, \infty] \times(\alpha-1, \infty)$,

$$
\left\|\Lambda^{s} \chi\left(2^{-q} \Lambda\right) \mathcal{R}_{\alpha}\right\|_{\mathcal{L}\left(L^{p}\right)} \lesssim 2^{q(s+1-\alpha)} .
$$

(2)Let $\mathcal{C}$ be a ring. Then there exists $\phi \in \mathcal{S}\left(\mathbb{R}^{d}\right)$ whose spectrum does not meet the origin such that

$$
\mathcal{R}_{\alpha} u=2^{q(d+1-\alpha)} \phi\left(2^{q} \cdot\right) * u
$$

for every $u$ with Fourier variable supported on $2^{q} \mathcal{C}$.

The following Lemma is useful in dealing with the commutator terms.

Lemma 3.2. Let $p \in[1, \infty]$ and $\delta \in(0,1)$.

(1) If $|x|^{\delta} \phi \in L^{1}, f \in \dot{B}_{p, \infty}^{\delta}$ and $g \in L^{\infty}$, then

$$
\|\phi *(f g)-f(\phi * g)\|_{L^{p}} \leq\left. C\|\| x\right|^{\delta} \phi\left\|_{L^{1}}\right\| f\left\|_{\dot{B}_{p, \infty}^{\delta}}\right\| g \|_{L^{\infty}} .
$$

In the case when $\delta=1$, we have

$$
\|\phi *(f g)-f(\phi * g)\|_{L^{p}} \leq C\|\| x \mid \phi\left\|_{L^{1}}\right\| \nabla f\left\|_{L^{p}}\right\| g \|_{L^{\infty}} .
$$

(2) If $|x|^{\delta} \phi \in L^{1}, f \in \dot{B}_{\infty, \infty}^{\delta}$ and $g \in L^{p}$, then

$$
\|\phi *(f g)-f(\phi * g)\|_{L^{p}} \leq\left. C\|\| x\right|^{\delta} \phi\left\|_{L^{1}}\right\| f\left\|_{\dot{B}_{\infty, \infty}^{\delta}}\right\| g \|_{L^{p}} .
$$

In the case when $\delta=1$, we have

$$
\|\phi *(f g)-f(\phi * g)\|_{L^{p}} \leq C\|\| x \mid \phi\left\|_{L^{1}}\right\| \nabla f\left\|_{L^{\infty}}\right\| g \|_{L^{p}} .
$$

The first part is proved in [1], so we just prove the second part of the above Lemma. 
Proof. By Minkowski's inequality, for any $p \in[1, \infty]$,

$$
\begin{aligned}
\| \phi & *(f g)-f(\phi * g) \|_{L^{p}} \\
& =\left[\int\left|\int \phi(z)(f(x)-f(x-z)) g(x-z) d z\right|^{p} d x\right]^{1 / p} \\
& \leq \int\left[\int|\phi(z)(f(x)-f(x-z)) g(x-z)|^{p} d x\right]^{1 / p} d z \\
& \leq \int \frac{\|f(\cdot)-f(\cdot-z)\|_{L^{\infty}}}{|z|^{\delta}}|z|^{\delta}|\phi(z)|\left(\int|g(x-z)|^{p} d x\right)^{1 / p} d z \\
& \leq\left\||x|^{\delta} \phi\right\|_{L^{1}} \sup _{|z|>0} \frac{\|f(\cdot)-f(\cdot-z)\|_{L^{\infty}}}{|z|^{\delta}}\|g\|_{L^{p}} \\
& \leq C\left\||x|^{\delta} \phi\right\|_{L^{1}}\|f\|_{\dot{B}_{\infty, \infty}^{\delta}}\|g\|_{L^{p}} .
\end{aligned}
$$

In the last inequality, we use the definition of $\dot{B}_{\infty, \infty}^{\delta}$.

The next Theorem concerns the crucial commutators involving $\mathcal{R}_{\alpha}$.

Theorem 3.3. Let $\alpha \in(0,1), \epsilon>0$ taken to be small enough, $u$ be a smooth divergence-free vector field of $\mathbb{R}^{d}$ and $\theta$ be a smooth scalar function. Then,

(1) For every $(s, p, q) \in(-1, \alpha-\sigma) \times[2, \infty] \times[1, \infty]$ and take $\epsilon>0$ satisfy $s+\sigma+\epsilon<\alpha$ we have

$$
\left\|\left[\mathcal{R}_{\alpha}, u \cdot \nabla\right] \theta\right\|_{B_{p, q}^{s}} \lesssim\|u\|_{\dot{B}_{p, \infty}^{1-\sigma-\epsilon}}\left(\|\theta\|_{B_{\infty, q}^{s+1+\sigma+\epsilon-\alpha}}+\|\theta\|_{L^{\infty}}\right) .
$$

(2) In the 2 dimensional case, if $u=\nabla^{\perp} \Delta^{-1} \Lambda^{\sigma}(\log (I d-\Delta))^{\gamma} \omega, \omega:=G+$ $\mathcal{R}_{\alpha} \theta$ and $0 \leq \sigma<\alpha<1$. Then for every $0<s<\alpha-\sigma$, taking arbitrary small $\epsilon>0$ such that $s+\sigma+\epsilon<\alpha$, we have

$$
\begin{aligned}
\left\|\left[\mathcal{R}_{\alpha}, u\right] \theta\right\|_{H^{s}} \lesssim & \|G\|_{L^{2}}\|\theta\|_{B_{\infty, 2}^{s+\sigma+\epsilon-\alpha}}+\|\theta\|_{L^{\infty}}\|\theta\|_{H^{s+1+\sigma+\epsilon-2 \alpha}} \\
& +\|G\|_{L^{2}}\|\theta\|_{L^{\frac{2}{1-\sigma}}}+\|\theta\|_{L^{2}}\|\theta\|_{L^{\frac{2}{1-\sigma}}} .
\end{aligned}
$$

Proof. (1) Due to Bony's decomposition we split the commutator term into three parts

$$
\begin{aligned}
{\left[\mathcal{R}_{\alpha}, u \cdot \nabla\right] \theta=} & \sum_{n \in \mathbb{N}}\left[\mathcal{R}_{\alpha}, S_{n-1} u \cdot \nabla\right] \Delta_{n} \theta+\sum_{n \in \mathbb{N}}\left[\mathcal{R}_{\alpha}, \Delta_{n} u \cdot \nabla\right] S_{n-1} \theta \\
& +\sum_{n \geq-1}\left[\mathcal{R}_{\alpha}, \Delta_{n} u \cdot \nabla\right] \widetilde{\Delta}_{n} \theta \\
= & I+I I+I I I
\end{aligned}
$$

For I, since for every $n \in \mathbb{N}$ the Fourier transform of $S_{n-1} u \Delta_{n} \theta$ is supported in a ring of size $2^{n}$, then from Theorem 3.1 and Lemma 3.2, we have 
for every $j \geq-1$

$$
\begin{aligned}
\left\|\Delta_{j} I\right\|_{L^{p}} & \lesssim \sum_{|n-j| \leq 4}\left\|\left[\phi_{n} *, S_{n-1} u \cdot \nabla\right] \Delta_{n} \theta\right\|_{L^{p}} \\
& \lesssim \sum_{|n-j| \leq 4} 2^{n(\sigma+\epsilon-\alpha)}\|u\|_{\dot{B}_{p, \infty}^{1-\sigma-\epsilon}} 2^{n}\left\|\Delta_{n} \theta\right\|_{L^{\infty}} \\
& \lesssim c_{j} 2^{-j s}\|u\|_{\dot{B}_{p, \infty}^{1-\sigma-\epsilon}}\|\theta\|_{B_{\infty}^{s+, q}}+\epsilon+1-\alpha
\end{aligned}
$$

where $\phi_{n}(x):=2^{n(d+1-\alpha)} \phi\left(2^{n} x\right)$ with $\phi \in \mathcal{S}$ and $\left(c_{j}\right)_{j \geq-1}$ with $\left\|c_{j}\right\|_{\ell^{q}}=1$.

Thus we obtain

$$
\|I\|_{B_{p, q}^{s}} \lesssim\|u\|_{\dot{B}_{p, \infty}^{1-\sigma-\epsilon}}\|\theta\|_{B_{\infty, q}^{s+\sigma+\epsilon+1-\alpha} .}
$$

For II, similar to I we have

$$
\begin{aligned}
& \left\|\Delta_{j} I I\right\|_{L^{p}} \\
& \quad \lesssim \sum_{|n-j| \leq 4, n \in \mathbb{N}}\left\|\left[\phi_{n} *, \Delta_{n} u \cdot \nabla\right] S_{n-1} \theta\right\|_{L^{p}} \\
& \quad \lesssim \sum_{|n-j| \leq 4} 2^{n(\sigma+\epsilon-\alpha)}\|u\|_{\dot{B}_{p, \infty}^{1-\sigma-\epsilon}}\left\|\nabla S_{n-1} \theta\right\|_{L^{\infty}} \\
& \quad \lesssim\|u\|_{\dot{B}_{p, \infty}^{1-\sigma-\epsilon}} 2^{-j s} \sum_{-1 \leq n^{\prime} \leq j+2} 2^{\left(n^{\prime}-j\right)(\alpha-\sigma-\epsilon-s)} 2^{n^{\prime}(s+\sigma+\epsilon-\alpha+1)}\left\|\Delta_{n^{\prime}} \theta\right\|_{L^{\infty}} .
\end{aligned}
$$

Thus using discrete Young's inequality we obtain

$$
\|I I\|_{B_{p, q}^{s}} \lesssim\|u\|_{\dot{B}_{p, \infty}^{1-\sigma-\epsilon}}\|\theta\|_{B_{\infty, q}^{s+\sigma+\epsilon+1-\alpha} .}
$$

For III, we further write

$$
\begin{aligned}
I I I & =\sum_{n \geq 0} \operatorname{div}\left[\mathcal{R}_{\alpha}, \Delta_{n} u\right] \widetilde{\Delta}_{n} \theta+\sum_{1 \leq i \leq n}\left[\partial_{i} \mathcal{R}_{\alpha}, \Delta_{-1} u^{i}\right] \widetilde{\Delta}_{-1} \theta \\
& =I I I^{1}+I I I^{2} .
\end{aligned}
$$

Considering Bernstein's inequality and Theorem 3.1, we deal with the term $I I I^{1}$ as follows

$$
\begin{aligned}
& \left\|\Delta_{j} I I I^{1}\right\|_{L^{p}} \\
& \quad \lesssim \sum_{n \geq j-3, n \geq 0}\left\|\Delta_{j} \operatorname{div} \mathcal{R}_{\alpha}\left(\Delta_{n} u \widetilde{\Delta}_{n} \theta\right)\right\|_{L^{p}}+\sum_{n \geq j-3, n \geq 0}\left\|\Delta_{j} \operatorname{div}\left(\Delta_{n} u \mathcal{R}_{\alpha} \widetilde{\Delta}_{n} \theta\right)\right\|_{L^{p}} \\
& \quad \lesssim \sum_{n \geq j-3}\left(2^{j(2-\alpha)}+2^{j} 2^{n(1-\alpha)}\right) 2^{-n(1-\sigma-\epsilon)}\left\|\Delta_{n} \Lambda^{1-\sigma-\epsilon} u\right\|_{L^{p}}\left\|\widetilde{\Delta}_{n} \theta\right\|_{L^{\infty}} \\
& \quad \lesssim\|u\|_{\dot{B}_{p, \infty}^{1-\sigma-\epsilon}} 2^{-j s} \sum_{n \geq j-4}\left(2^{(j-n)(s+2-\alpha)}+2^{(j-n)(s+1)}\right) 2^{n(s+1+\sigma+\epsilon-\alpha)}\left\|\Delta_{n} \theta\right\|_{L^{\infty}} .
\end{aligned}
$$

Thus we obtain for every $s>-1$

$$
\left\|I I I^{1}\right\|_{B_{p, q}^{s}} \lesssim\|u\|_{\dot{B}_{p, \infty}^{1-\sigma-\epsilon}}\|\theta\|_{B_{\infty, q}^{s+1+\sigma+\epsilon-\alpha}}
$$


Choosing a suitable function $\chi \in \mathcal{D}\left(\mathbb{R}^{d}\right)$, we have

$$
I I I^{2}=\sum_{1 \leq i \leq n}\left[\partial_{i} \mathcal{R}_{\alpha} \chi(D), \Delta_{-1} u^{i}\right] \widetilde{\Delta}_{-1} \theta .
$$

By Theorem 3.1, we know that $\partial_{i} \mathcal{R}_{\alpha} \chi(D)$ is a convolution operator with kernel $h$ satisfying

$$
|h(x)| \leq C(1+|x|)^{-d-2+\alpha}, \quad \text { for all } x \in \mathbb{R}^{d} .
$$

Next, from the fact that $\Delta_{j} I I I^{2}=0$ for every $j \geq 3$ and using Lemma 3.2 , we have

$$
\begin{aligned}
\left\|I I I^{2}\right\|_{B_{p, q}^{s}} & \lesssim\left\|\left[h *, \Delta_{-1} u\right] \widetilde{\Delta}_{-1} \theta\right\|_{L^{p}} \\
& \lesssim\left\||x|^{1-\sigma-\epsilon} h\right\|_{L^{1}}\left\|\Delta_{-1} u\right\|_{\dot{B}_{p, \infty}^{1-\sigma-\epsilon}}\left\|\widetilde{\Delta}_{-1} \theta\right\|_{L^{\infty}} \\
& \lesssim\|u\|_{\dot{B}_{p, \infty}^{1-\sigma-\epsilon}}\|\theta\|_{L^{\infty}} .
\end{aligned}
$$

Hence, the proof of the first part is complete.

(2) As in the first part, we can get the following equality by Bony's decomposition.

$$
\begin{aligned}
{\left[\mathcal{R}_{\alpha}, u\right] \theta } & =\sum_{n \in \mathbb{N}}\left[\mathcal{R}_{\alpha}, S_{n-1} u\right] \Delta_{n} \theta+\sum_{n \in \mathbb{N}}\left[\mathcal{R}_{\alpha}, \Delta_{n} u\right] S_{n-1} \theta+\sum_{n \geq-1}\left[\mathcal{R}_{\alpha}, \Delta_{n} u\right] \widetilde{\Delta}_{n} \theta \\
& =I+I I+I I I .
\end{aligned}
$$

For brevity, let $P(\Lambda):=\Lambda^{\sigma}(\log (\operatorname{Id}-\Delta))^{\gamma}$. For I, denote $I_{n}:=$ $\left[\mathcal{R}_{\alpha}, S_{n-1} u\right] \Delta_{n} \theta$. Since for each $n \in \mathbb{N}$ the Fourier transform of $S_{n-1} u \Delta_{n} \theta$ is supported in a ring of size $2^{n}$, from Theorem 3.1 there exists $\phi \in \mathcal{S}\left(\mathbb{R}^{2}\right)$ whose spectrum is away from the origin such that

$$
I_{n}=\left[\phi_{n} *, S_{n-1} \nabla^{\perp} \Delta^{-1} P(\Lambda) G\right] \Delta_{n} \theta+\left[\phi_{n} *, S_{n-1} \nabla^{\perp} \Delta^{-1} P(\Lambda) \mathcal{R}_{\alpha} \theta\right] \Delta_{n} \theta,
$$

where $\phi_{n}(x):=2^{n(d+1-\alpha)} \phi\left(2^{n} x\right)$. Using Lemma 3.2 and Theorem 3.1, we obtain

$$
\begin{aligned}
\left\|I_{n}\right\|_{L^{2}} \lesssim & \left\||x|^{1-\sigma-\epsilon} \phi_{n}\right\|_{L^{1}}\left\|\Lambda^{1-\sigma-\epsilon} S_{n-1} \nabla^{\perp} \Delta^{-1} P(\Lambda) G\right\|_{L^{2}}\left\|\Delta_{n} \theta\right\|_{L^{\infty}} \\
& +\left\||x|^{1-\sigma-\epsilon} \phi_{n}\right\|_{L^{1}}\left\|\Lambda^{1-\sigma-\epsilon} S_{n-1} \nabla^{\perp} \Delta^{-1} P(\Lambda) \mathcal{R}_{\alpha} \theta\right\|_{L^{\infty}}\left\|\Delta_{n} \theta\right\|_{L^{2}} \\
\lesssim & 2^{n(\sigma+\epsilon-\alpha)}\left\||x|^{1-\sigma-\epsilon} \phi\right\|_{L^{1}}\|G\|_{L^{2}}\left\|\Delta_{n} \theta\right\|_{L^{\infty}} \\
& +2^{n(\sigma+\epsilon-\alpha)} 2^{n(1-\alpha)}\left\||x|^{1-\sigma-\epsilon} \phi\right\|_{L^{1}}\|\theta\|_{L^{\infty}}\left\|\Delta_{n} \theta\right\|_{L^{2}} \\
\lesssim & 2^{n(\sigma+\epsilon-\alpha)}\|G\|_{L^{2}}\left\|\Delta_{n} \theta\right\|_{L^{\infty}}+2^{n(\sigma+\epsilon-\alpha)} 2^{n(1-\alpha)}\|\theta\|_{L^{\infty}}\left\|\Delta_{n} \theta\right\|_{L^{2}} .
\end{aligned}
$$

Thus, we can obtain

$$
\|I\|_{H^{s}} \lesssim\|G\|_{L^{2}}\|\theta\|_{B_{\infty, 2}^{s+\sigma+\epsilon-\alpha}}+\|\theta\|_{L^{\infty}}\|\theta\|_{H^{s+\sigma+\epsilon+1-2 \alpha}} .
$$

For II, denote $I I_{n}:=\left[\mathcal{R}_{\alpha}, \Delta_{n} u\right] S_{n-1} \theta$. As in I, we have

$$
I I_{n}=\left[\phi_{n} *, \Delta_{n} \Delta^{-1} \nabla^{\perp} P(\Lambda) G\right] S_{n-1} \theta+\left[\phi_{n} *, \Delta_{n} \Delta^{-1} \nabla^{\perp} P(\Lambda) \mathcal{R}_{\alpha} \theta\right] S_{n-1} \theta
$$


By Lemma 3.2, we get

$$
\begin{aligned}
\left\|I I_{n}\right\|_{L^{2}} \lesssim & \left\||x|^{1-\sigma-\epsilon} \phi_{n}\right\|_{L^{1}}\left\|\Lambda^{1-\sigma-\epsilon} \Delta_{n} \nabla^{\perp} \Delta^{-1} P(\Lambda) G\right\|_{L^{2}}\left\|S_{n-1} \theta\right\|_{L^{\infty}} \\
& +\left\||x|^{1-\sigma-\epsilon} \phi_{n}\right\|_{L^{1}}\left\|\Lambda^{1-\sigma-\epsilon} \Delta_{n} \nabla^{\perp} \Delta^{-1} P(\Lambda) \mathcal{R}_{\alpha} \theta\right\|_{L^{2}}\left\|S_{n-1} \theta\right\|_{L^{\infty}} \\
\lesssim & 2^{n(\sigma+\epsilon-\alpha)}\|G\|_{L^{2}}\left\|S_{n-1} \theta\right\|_{L^{\infty}}+2^{n(\sigma+\epsilon-\alpha)} 2^{n(1-\alpha)}\left\|\Delta_{n} \theta\right\|_{L^{2}}\|\theta\|_{L^{\infty}}
\end{aligned}
$$

Hence, by discrete Young's inequality, we have

$$
\|I I\|_{H^{s}} \lesssim\|G\|_{L^{2}}\|\theta\|_{B_{\infty, 2}^{s+\sigma+\epsilon-\alpha}}+\|\theta\|_{L^{\infty}}\|\theta\|_{H^{s+1+\sigma+\epsilon-2 \alpha}} .
$$

We further write III as follows

$$
\begin{aligned}
I I I & =\sum_{n \geq 0} \mathcal{R}_{\alpha}\left(\Delta_{n} u \widetilde{\Delta}_{n} \theta\right)+\sum_{n \geq 0} \Delta_{n} u \mathcal{R}_{\alpha} \widetilde{\Delta}_{n} \theta+\left[\mathcal{R}_{\alpha}, \Delta_{-1} u\right] \widetilde{\Delta}_{-1} \theta \\
& =I I I^{1}+I I I^{2}+I I I^{3} .
\end{aligned}
$$

First, we note that for every $n \geq 0$

$$
\left\|\Delta_{n} u\right\|_{L^{2}} \lesssim 2^{n(\sigma+\epsilon-1)}\left\|\Delta_{n} G\right\|_{L^{2}}+2^{n(\sigma+\epsilon-\alpha)}\left\|\Delta_{n} \theta\right\|_{L^{2}} .
$$

Then by a direct computation we have $2^{j s}\left\|\Delta_{j} I I I^{1}\right\|_{L^{2}}$

$\lesssim 2^{j(s+1-\alpha)} \sum_{n \geq j-3, n \geq 0}\left\|\Delta_{n} u\right\|_{L^{2}}\left\|\widetilde{\Delta}_{n} \theta\right\|_{L^{\infty}}$

$\lesssim 2^{j(s+1-\alpha)} \sum_{n \geq j-3}\left(2^{n(\sigma+\epsilon-1)}\left\|\Delta_{n} G\right\|_{L^{2}}+2^{n(\sigma+\epsilon-\alpha)}\left\|\Delta_{n} \theta\right\|_{L^{2}}\right)\left\|\widetilde{\Delta}_{n} \theta\right\|_{L^{\infty}}$

$\lesssim \sum_{n \geq j-4} 2^{(j-n)(s+1-\alpha)}\left(2^{n(s+\sigma+\epsilon-\alpha)}\left\|\Delta_{n} \theta\right\|_{L^{\infty}}\|G\|_{L^{2}}\right.$

$$
\left.+2^{n(s+\sigma+\epsilon+1-2 \alpha)}\left\|\Delta_{n} \theta\right\|_{L^{2}}\|\theta\|_{L^{\infty}}\right) .
$$

Thus discrete Young's inequality yields

$$
\left\|I I I^{1}\right\|_{H^{s}} \lesssim\|G\|_{L^{2}}\|\theta\|_{B_{\infty, 2}^{s+\sigma+\epsilon-\alpha}}+\|\theta\|_{L^{\infty}}\|\theta\|_{H^{s+1+\sigma+\epsilon-2 \alpha}} .
$$

For $I I I^{2}$, similar to $I I I^{1}$ and using Theorem 3.1 we have $2^{j s}\left\|\Delta_{j} I I I^{2}\right\|_{L^{2}}$

$$
\begin{aligned}
& \lesssim 2^{j s} \sum_{n \geq j-3, n \geq 0}\left\|\Delta_{n} u\right\|_{L^{2}}\left\|\mathcal{R}_{\alpha} \widetilde{\Delta}_{n} \theta\right\|_{L^{\infty}} \\
& \lesssim 2^{j s} \sum_{n \geq j-3}\left(2^{n(\sigma+\epsilon-1)}\left\|\Delta_{n} G\right\|_{L^{2}}+2^{n(\sigma+\epsilon-\alpha)}\left\|\Delta_{n} \theta\right\|_{L^{2}}\right) 2^{n(1-\alpha)}\left\|\widetilde{\Delta}_{n} \theta\right\|_{L^{\infty}} \\
& \lesssim \sum_{n \geq j-4} 2^{(j-n) s}\left(2^{n(s+\sigma+\epsilon-\alpha)}\left\|\Delta_{n} \theta\right\|_{L^{\infty}}\|G\|_{L^{2}}\right. \\
& \left.+2^{n(s+\sigma+\epsilon+1-2 \alpha)}\left\|\Delta_{n} \theta\right\|_{L^{2}}\|\theta\|_{L^{\infty}}\right) .
\end{aligned}
$$

Using convolution inequality, we obtain

$$
\left\|I I I^{2}\right\|_{H^{s}} \lesssim\|G\|_{L^{2}}\|\theta\|_{B_{\infty, 2}^{s+\sigma+\epsilon-\alpha}}+\|\theta\|_{L^{\infty}}\|\theta\|_{H^{s+1+\sigma+\epsilon-2 \alpha}} .
$$


For $I I I^{3}$, since $\Delta_{j} I I I^{3}=0$ for every $j \geq 3$, then from Bernstein's inequality we immediately have

$$
\begin{aligned}
\left\|I I I^{3}\right\|_{H^{s}} & \lesssim\left\|\mathcal{R}_{\alpha}\left(\Delta_{-1} u \widetilde{\Delta}_{-1} \theta\right)\right\|_{L^{2}}+\left\|\Delta_{-1} u \mathcal{R}_{\alpha} \widetilde{\Delta}_{-1} \theta\right\|_{L^{2}} \\
& \lesssim\left\|\Delta_{-1} u \widetilde{\Delta}_{-1} \theta\right\|_{L^{2}}+\left\|\Delta_{-1} u \mathcal{R}_{\alpha} \widetilde{\Delta}_{-1} \theta\right\|_{L^{2}} \\
& \lesssim\|G\|_{L^{2}}\|\theta\|_{\frac{2}{1-\sigma}}+\|\theta\|_{L^{2}}\|\theta\|_{L^{\frac{2}{1-\sigma}}}
\end{aligned}
$$

Here, we used the following fact

$$
\begin{aligned}
\left\|\Delta_{-1} u \widetilde{\Delta}_{-1} \theta\right\|_{L^{2}} & \lesssim\left\|\Delta_{-1} u\right\|_{L^{q_{1}}}\left\|\widetilde{\Delta}_{-1} \theta\right\|_{L^{p_{2}}} \\
& \lesssim\left\|\Delta_{-1} \Lambda^{\sigma-1+\epsilon} \omega\right\|_{L^{q_{1}}}\left\|\widetilde{\Delta}_{-1} \theta\right\|_{L^{p_{2}}} \\
& \lesssim\left\|\Delta_{-1} \omega\right\|_{L^{p_{1}}}\left\|\widetilde{\Delta}_{-1} \theta\right\|_{L^{p_{2}}} \\
& \lesssim\left\|\Delta_{-1} G\right\|_{L^{p_{1}}}\left\|\widetilde{\Delta}_{-1} \theta\right\|_{L^{p_{2}}}+\left\|\Delta_{-1} \mathcal{R}_{\alpha} \theta\right\|_{L^{p_{1}}}\left\|\widetilde{\Delta}_{-1} \theta\right\|_{L^{p_{2}}} \\
& \lesssim\|G\|_{L^{p_{1}}}\|\theta\|_{L^{p_{2}}}+\|\theta\|_{L^{p_{1}}}\|\theta\|_{L^{p_{2}}},
\end{aligned}
$$

where $\frac{1}{q_{1}}+\frac{1}{p_{2}}=\frac{1}{2}$ and $\frac{1}{p_{1}}+\frac{1}{p_{2}}=\frac{1}{2}+\frac{1-\sigma}{2}$. And taking $p_{1}=2, p_{2}=\frac{2}{1-\sigma}$ in our deduction. From all the above statements, we can obtain our conclusion.

\section{Some priori estimates}

First we need to introduce some notations. Let $G:=\omega-\mathcal{R}_{\alpha} \theta$. Considering the vorticity equation

$$
\partial_{t} \omega+u \cdot \nabla \omega+\Lambda^{\alpha} \omega=\partial_{1} \theta
$$

and the acting of $\mathcal{R}_{\alpha}$ on the temperature equation

$$
\partial_{t} \mathcal{R}_{\alpha} \theta+u \cdot \nabla \mathcal{R}_{\alpha} \theta+\Lambda^{\beta} \mathcal{R}_{\alpha} \theta=-\left[\mathcal{R}_{\alpha}, u \cdot \nabla\right] \theta,
$$

we directly have

$$
\partial_{t} G+u \cdot \nabla G+\Lambda^{\alpha} G=\left[\mathcal{R}_{\alpha}, u \cdot \nabla\right] \theta+\Lambda^{\beta} \mathcal{R}_{\alpha} \theta
$$

\subsection{Estimation of $\|G\|_{L^{2}}$}

We present a Lemma that is proved in [6], for it is useful in our proof.

Lemma 4.1. Let $(\omega, \theta)$ be a smooth solution of the system (1.1). Then for every $m \in[2, \infty]$ and $t \in \mathbb{R}^{+}$

$$
\|\theta\|_{L_{t}^{m} \dot{H} \frac{\beta}{m}} \lesssim\left\|\theta_{0}\right\|_{L^{2}}
$$

and for $p \in[1, \infty]$

$$
\|\theta(t)\|_{L^{p}} \leq\left\|\theta_{0}\right\|_{L^{p}}
$$

The following is our estimation about $\|G\|_{L^{2}}$.

Theorem 4.2. Consider (1.1) with $\sigma=0$ and $\gamma \geq 0$. Assume that $\left(\omega_{0}, \theta_{0}\right)$ satisfies the conditions in Theorem 1.1. Let $(\omega, \theta)$ be the corresponding solution of (1.1), $G$ is defined as above. Then if $\alpha, \beta$ satisfies $(\alpha, \beta) \in\left(\frac{3}{4}, 1\right) \times(1-$ $\alpha, \min \{3 \alpha-2,2-2 \alpha\})]$. 
Then the following inequality holds true

$$
\|G(t)\|_{L^{2}}^{2}+\int_{0}^{t}\|G(\tau)\|_{\dot{H}^{\frac{\alpha}{2}}}^{2} d \tau \leq B(t)
$$

where $B(t)$ is a smooth function of $t$ depending on the initial data only.

Proof. Multiplying $G$ to Eq. (4.1) and integrating with spatial variable, we obtain

$$
\begin{aligned}
\frac{1}{2} \frac{d}{d t}\|G\|_{L^{2}}^{2}+\left\|\Lambda^{\frac{\alpha}{2}} G\right\|_{L^{2}}^{2} & =\int\left[\mathcal{R}_{\alpha}, u \cdot \nabla\right] \theta G d x+\int \Lambda^{\beta-\alpha} \partial_{1} \theta G d x \\
& =I+I I
\end{aligned}
$$

For I, we have

$$
\begin{aligned}
|I| & =\left|\int \operatorname{div}\left[\mathcal{R}_{\alpha}, u\right] \theta G d x\right| \\
& \leq\left\|\Lambda^{\frac{\alpha}{2}} G\right\|_{L^{2}}\left\|\left[\mathcal{R}_{\alpha}, u\right] \theta\right\|_{\dot{H}^{1-\frac{\alpha}{2}}}
\end{aligned}
$$

Choosing $p_{3}>\frac{2}{\frac{3}{2} \alpha-1-\epsilon}$ and using Theorem 3.3, we obtain

$$
\begin{aligned}
& \left\|\left[\mathcal{R}_{\alpha}, u\right] \theta\right\|_{\dot{H}^{1-\frac{\alpha}{2}}} \\
& \quad \lesssim\|G\|_{L^{2}}\|\theta\|_{B_{\infty, 2}^{1+\epsilon-\frac{3}{2} \alpha}}+\|\theta\|_{L^{\infty}}\|\theta\|_{H^{2+\epsilon-\frac{5}{2} \alpha}}+\|G\|_{L^{2}}\|\theta\|_{L^{2}}+\|\theta\|_{L^{2}}\|\theta\|_{L^{2}} \\
& \quad \lesssim\|G\|_{L^{2}}\|\theta\|_{L^{p_{3}}}+\|\theta\|_{L^{\infty}}\|\theta\|_{H^{2+\epsilon-\frac{5}{2} \alpha}}+\|G\|_{L^{2}}\|\theta\|_{L^{2}}+\|\theta\|_{L^{2}}\|\theta\|_{L^{2}}
\end{aligned}
$$

where $\epsilon>0$ is an arbitrary small number. For II, choosing $s_{1} \in\left[0, \frac{\alpha}{2}\right]$, we have

$$
|I I| \leq\left\|\Lambda^{s_{1}} G\right\|_{L^{2}}\left\|\Lambda^{1+\beta-\alpha-s_{1}} \theta\right\|_{L^{2}} .
$$

From above statements, we can obtain

$$
\begin{aligned}
& \frac{1}{2} \frac{d}{d t}\|G\|_{L^{2}}^{2}+\left\|\Lambda^{\frac{\alpha}{2}} G\right\|_{L^{2}}^{2} \\
& \quad \lesssim\|G\|_{L^{2}}\|\theta\|_{L^{p_{3}}}+\|\theta\|_{L^{\infty}}\|\theta\|_{H^{2+\epsilon-\frac{5}{2}} \alpha} \\
& \quad+\|G\|_{L^{2}}\|\theta\|_{L^{2}}+\|\theta\|_{L^{2}}\|\theta\|_{L^{2}}+\left\|\Lambda^{s_{1}} G\right\|_{L^{2}}\|\theta\|_{\dot{H}^{1+\beta-\alpha-s_{1}}}
\end{aligned}
$$

From interpolation inequality and Young's inequality, we obtain

$$
\begin{aligned}
& \|\theta\|_{\dot{H}^{1+\beta-\alpha-s_{1}}}\|G\|_{\dot{H}^{s_{1}}} \\
& \quad \leq C\|\theta\|_{\dot{H}^{1+\beta-\alpha-s_{1}}}\|G\|_{\dot{H}^{\frac{\alpha}{2}}}^{\frac{2 s_{1}}{\alpha}}\|G\|_{L^{2}}^{1-\frac{2 s_{1}}{\alpha}} \\
& \quad \leq C\|\theta\|_{\dot{H}^{1+\beta-\alpha-s_{1}}}^{2}+C\|G\|_{L^{2}}^{2}+\frac{1}{4}\|G\|_{\dot{H}^{\frac{\alpha}{2}}}^{2} .
\end{aligned}
$$

Using Young's inequality and the above inequality, we have

$$
\begin{aligned}
& \frac{d}{d t}\|G\|_{L^{2}}^{2}+\left\|\Lambda^{\frac{\alpha}{2}} G\right\|_{L^{2}}^{2} \\
& \quad \leq C\|G\|_{L^{2}}^{2}+C\left\|\theta_{0}\right\|_{L^{2} \cap L^{\infty}}+C\|\theta\|_{H^{2+\epsilon-\frac{5}{2} \alpha}}^{2}+C\|\theta\|_{\dot{H}^{1+\beta-\alpha-s_{1}}}^{2}
\end{aligned}
$$


Gronwall's inequality thus leads to

$$
\begin{aligned}
& \|G(t)\|_{L^{2}}^{2}+\int_{0}^{t}\left\|\Lambda^{\frac{\alpha}{2}} G(\tau)\right\|_{L^{2}}^{2} d \tau \\
& \quad \leq C e^{C t}\left(t+\|\theta\|_{L_{t}^{2} H^{2+\epsilon-\frac{5}{2} \alpha}}^{2}+\|\theta\|_{L_{t}^{2} \dot{H}^{1+\beta-\alpha-s_{1}}}^{2}\right) .
\end{aligned}
$$
we have

If $\frac{3}{4}<\alpha \leq \frac{4}{5}$, for $1-\alpha<\beta \leq 3 \alpha-2$, then clearly for $\epsilon>0$ small enough

$$
\begin{aligned}
& 0 \leq 1+\beta-\frac{3}{2} \alpha \leq \frac{\beta}{2}, \\
& 0 \leq 2+\epsilon-\frac{5}{2} \alpha \leq \frac{\beta}{2},
\end{aligned}
$$

Using Lemma 4.1 and interpolation inequality we easily get

$$
\|\theta\|_{L_{t}^{2} H^{2-\frac{5}{2} \alpha+\epsilon}}^{2}+\|\theta\|_{L_{t}^{2} \dot{H}^{1+\beta-\frac{3}{2} \alpha}}^{2} \lesssim 1+t
$$
then

If $\frac{4}{5}<\alpha<1$, we choose $s_{1}=2-2 \alpha \in\left(0, \frac{\alpha}{2}\right)$, and for $1-\alpha<\beta \leq 2-2 \alpha$,

$$
0 \leq \beta-1+\alpha \leq \frac{\beta}{2}
$$

Using Lemma 4.1 and interpolation inequality we get

$$
\|\theta\|_{L_{t}^{2} \dot{H}^{\beta-1+\alpha}}^{2}+\|\theta\|_{L_{t}^{2} H^{2-\frac{5}{2} \alpha+\epsilon}}^{2} \lesssim 1+t .
$$

Hence, the proof is complete.

\subsection{Estimation of $\|G\|_{L^{q}}$ for $q$ in suitable range}

This subsection presents the estimate of $\|G\|_{L^{q}}$ for $q$ in some suitable range. Before the main theorem, we need two Lemmas $[6,17]$.

Lemma 4.3. Suppose that $s \in[0,1]$, and $f,(-\Delta)^{s} f \in L^{p}\left(\mathbb{R}^{2}\right), p \geq 2$. Then

$$
\int_{\mathbb{R}^{2}}|f|^{p-2} f(-\Delta)^{s} f d x \geq \frac{2}{p} \int_{\mathbb{R}^{2}}\left((-\Delta)^{\frac{s}{2}}|f|^{\frac{p}{2}}\right)^{2} d x
$$

Lemma 4.4. Let $\gamma \in[2, \infty), s \in(0,1), \alpha \in\left(\frac{\gamma-4}{\gamma-2}, 2\right)$. Then for every smooth function $f$ we have

$$
\left\||f|^{\gamma-2} f\right\|_{\dot{H}^{s}} \lesssim\|f\|_{L^{\frac{2 \gamma}{2-\alpha}}}^{\gamma-2}\|f\|_{\dot{H}^{s+\left(\frac{d}{2}-\frac{d}{\gamma}\right)(2-\alpha)}} .
$$

The main theorem in this subsection can be stated as follows.

Theorem 4.5. Consider (1.1) with $\sigma=0$ and $\gamma \geq 0$. Assume that $\left(\omega_{0}, \theta_{0}\right)$ satisfies the conditions in Theorem 1.1. Let $(\omega, \theta)$ be the corresponding solution of (1.1), $G$ is defined as in (4.1). If $\alpha, \beta$ satisfies

$$
(\alpha, \beta) \in\left(\frac{9 q-12}{8 q-8}, 1\right) \times\left(1-\alpha, \min \left\{2-2 \alpha, \frac{5 q-4}{3 q-4} \alpha-2, \frac{1-\alpha}{\frac{4}{\alpha}\left(1-\frac{1}{q}\right)-2}\right\}\right)
$$


for some $q \in\left[2, \frac{20}{9}\right)$. Then for every $\widetilde{q} \in[2, q]$, we have for every $t \in \mathbb{R}^{+}$

$$
\|G(t)\|_{L^{\tilde{q}}}^{\widetilde{q}}+\int_{0}^{t}\|G(\tau)\|_{L^{\frac{2 \tilde{q}}{2-\alpha}}}^{\widetilde{q}} d \tau \leq B(t) .
$$

Proof. Multiplying (4.1) by $|G|^{q-2} G$ and integrating in the spatial variable we obtain for every $s_{2}, s_{3} \in\left(0, \frac{\alpha}{2}\right]\left(s_{3} \leq s_{2}\right.$ and both will be chosen later $)$

$$
\begin{aligned}
& \frac{1}{q} \frac{d}{d t}\|G(t)\|_{L^{q}}^{q}+\int_{\mathbb{R}^{2}} \Lambda^{\alpha} G|G|^{q-2} G(t) d x \\
& \quad \leq \int_{\mathbb{R}^{2}} \operatorname{div}\left[\mathcal{R}_{\alpha}, u\right] \theta|G|^{q-2} G(t) d x+\int_{\mathbb{R}^{2}} \Lambda^{\beta-\alpha} \partial_{1} \theta|G|^{q-2} G(t) d x \\
& \quad \leq\left\|\left[\mathcal{R}_{\alpha}, u\right] \theta(t)\right\|_{\dot{H}^{1-s_{2}}}\left\||G|^{q-2} G(t)\right\|_{\dot{H}^{s_{2}}}+\|\theta(t)\|_{\dot{H}^{1+\beta-\alpha-s_{3}}}\left\||G|^{q-2} G(t)\right\|_{\dot{H}^{s_{3}}} .
\end{aligned}
$$

Lemma 4.3 and continuous embedding $\dot{H}^{\frac{\alpha}{2}} \hookrightarrow L^{\frac{4}{2-\alpha}}$ lead to

$$
\int_{\mathbb{R}^{2}} \Lambda^{\alpha} G|G|^{q-2} G(t) d x \gtrsim\|G\|_{L^{\frac{2 q}{2-\alpha}}}^{q} .
$$

Using Lemma 4.4 we obtain

$$
\left\||G|^{q-2} G\right\|_{\dot{H}^{s_{i}}} \lesssim\|G\|_{L^{\frac{2 q}{2-\alpha}}}^{q-2}\|G\|_{\dot{H}^{s_{i}+\left(1-\frac{2}{q}\right)(2-\alpha)}}, \quad i=2,3 .
$$

From the above statements, we have

$$
\begin{aligned}
& \frac{d}{d t}\|G(t)\|_{L^{q}}^{q}+\|G\|_{L^{\frac{2 q}{2-\alpha}}}^{q} \\
& \quad \lesssim\left\|\left[\mathcal{R}_{\alpha}, u\right] \theta(t)\right\|_{\dot{H}^{1-s_{2}}}\|G(t)\|_{\dot{H}^{s_{2}+\left(1-\frac{2}{q}\right)(2-\alpha)}}\|G(t)\|_{L^{\frac{2 q}{2-\alpha}}}^{q-2} \\
& \quad+\|\theta(t)\|_{\dot{H}^{1+\beta-\alpha-s_{3}}}\|G(t)\|_{\dot{H}^{s_{3}+\left(1-\frac{2}{q}\right)(2-\alpha)}}\|G(t)\|_{L^{\frac{2 q}{2-\alpha}}}^{q-2}
\end{aligned}
$$

Then we choose $s_{2}$ such that $s_{2}+\left(1-\frac{2}{q}\right)(2-\alpha)=\frac{\alpha}{2}$ which calls for $s_{2}=\frac{\alpha}{2}-\left(1-\frac{2}{q}\right)(2-\alpha) \in\left(0, \frac{\alpha}{2}\right]$, this is plausible if $\alpha \in\left(\frac{4 q-8}{3 q-4}, 1\right)$ for $q \in[2,4)$. Since $s_{3} \leq s_{2}$ by interpolation we have

$$
\begin{aligned}
\|G(t)\|_{\dot{H}^{s_{3}+\left(1-\frac{2}{q}\right)(2-\alpha)}} & \lesssim\|G(t)\|_{\dot{H}^{\frac{\alpha}{2}}}^{\delta}\|G(t)\|_{L^{2}}^{1-\delta} \\
& \lesssim B(t)\|G(t)\|_{\dot{H}^{\frac{\alpha}{2}}}^{\delta},
\end{aligned}
$$

where $\delta:=\frac{2}{\alpha}\left(s_{3}+\left(1-\frac{2}{q}\right)(2-\alpha)\right)$. Form the definition, we know that $\delta \leq 1$. Also noting that if $\alpha \in\left(\frac{6 q-8}{5 q-4}, 1\right)$, we have $1-s_{2} \in(0, \alpha)$, then form Theorem 3.3 and estimation of $\|G\|_{L^{2}}$, we further get

$$
\begin{aligned}
& \left\|\left[\mathcal{R}_{\alpha}, u\right] \theta(t)\right\|_{H^{1-s_{2}}} \\
& \quad \lesssim\|G(t)\|_{L^{2}}\|\theta\|_{B_{\infty, 2}^{1-s_{2}-\alpha+\epsilon}}+\|\theta(t)\|_{L^{\infty}}\|\theta(t)\|_{H^{2-s_{2}-2 \alpha+\epsilon}} \\
& \quad+\|G(t)\|_{L^{2}}\|\theta(t)\|_{L^{2}}+\|\theta(t)\|_{L^{2}}\|\theta\|_{L^{2}} .
\end{aligned}
$$

Since $\alpha>\frac{2}{3}$ and $2 \leq q<\frac{4-2 \alpha}{3-\frac{5}{2} \alpha}$, we know that $1-s_{2}-\alpha+\epsilon<0$. Hence, we further get

$$
\left\|\left[\mathcal{R}_{\alpha}, u\right] \theta(t)\right\|_{H^{1-s_{2}}} \lesssim B(t)+\|\theta(t)\|_{H^{2-s_{2}-2 \alpha+\epsilon}} .
$$


Therefore we further have

$$
\begin{aligned}
& \frac{d}{d t}\|G(t)\|_{L^{q}}^{q}+\|G(t)\|_{L^{\frac{2 q}{2-\alpha}}}^{q} \\
& \quad \lesssim\left(B(t)+\|\theta(t)\|_{H^{2-2 \alpha-s_{2}+\epsilon}}\right)\|G(t)\|_{L^{\frac{2 q}{2-\alpha}}}^{q-2}\|G(t)\|_{\dot{H}^{\frac{\alpha}{2}}} \\
& \quad+B(t)\|\theta(t)\|_{\dot{H}^{1+\beta-\alpha-s_{3}}}\|G(t)\|_{L^{\frac{2 q}{2-\alpha}}}^{q-2}\|G(t)\|_{\dot{H}^{\frac{\alpha}{2}}}^{\delta} .
\end{aligned}
$$

Using Young inequality as follows

$$
\left|A_{1} A_{2} A_{3}\right| \leq C_{1}\left|A_{1}\right|^{\frac{2 q}{4-q \delta}}+C_{2}\left|A_{2}\right|^{\frac{2}{\delta}}+\frac{c}{4}\left|A_{3}\right|^{\frac{q}{q-2}}, \quad \text { for all } \delta \in(0,1]
$$

For $\frac{2 q}{4-\delta q} \geq 2$, we have

$$
\begin{aligned}
& \frac{d}{d t}\|G(t)\|_{L^{q}}^{q}+\|G(t)\|_{L^{\frac{2 q}{2-\alpha}}}^{q} \\
& \quad \lesssim B(t)+\|\theta(t)\|_{H^{2-2 \alpha-s_{2}+\epsilon}}^{\frac{2 q}{4-q}}+\|G(t)\|_{\dot{H}^{\frac{\alpha}{2}}}^{2}+B(t)\|\theta(t)\|_{\dot{H}^{1+\beta-\alpha-s_{3}}}^{\frac{2 q}{4-\delta q}},
\end{aligned}
$$

For other cases, we have

$$
\begin{aligned}
& \frac{d}{d t}\|G(t)\|_{L^{q}}^{q}+\|G(t)\|_{L^{\frac{2 q}{2-\alpha}}}^{q} \\
& \quad \lesssim B(t)+\|\theta(t)\|_{H^{2-2 \alpha-s_{2}+\epsilon}}^{\frac{2 q}{4-q}}+\|G(t)\|_{\dot{H}^{\frac{\alpha}{2}}}^{2}+\|\theta(t)\|_{\dot{H}^{1+\beta-\alpha-s_{3}}}^{2} .
\end{aligned}
$$

Integrating in time yields

$$
\begin{aligned}
& \|G(t)\|_{L^{q}}^{q}+\int_{0}^{t}\|G(\tau)\|_{L^{\frac{2 q}{2-\alpha}}}^{q} d \tau
\end{aligned}
$$

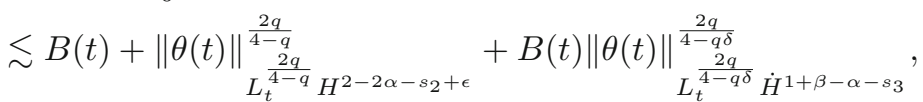

for $\frac{2 q}{4-q \delta} \geq 2$, and

$$
\begin{aligned}
& \|G(t)\|_{L^{q}}^{q}+\int_{0}^{t}\|G(\tau)\|_{L^{\frac{2 q}{2-\alpha}}}^{q} d \tau \\
& \lesssim B(t)+\|\theta(t)\|_{L_{t}^{\frac{2 q}{4-q}} H^{2-2 \alpha-s_{2}+\sigma+\epsilon}}^{\frac{2 q}{4-q}}+\|\theta(t)\|_{L_{t}^{2} \dot{H}^{1+\beta-\alpha-s_{3}}}^{2},
\end{aligned}
$$

for other cases. In the above calculus we use the conclusion of Theorem 4.2, so the range of $\alpha$ and $\beta$ must satisfy the conditions in Theorem 4.2.

Let $q \in\left[2, \frac{20}{9}\right)$. If $\alpha \in\left(\frac{9 q-12}{8 q-8}, \frac{8 q-8}{7 q-4}\right]$, we choose $s_{3}=s_{2}=\frac{3 q-4}{2 q} \alpha+\frac{4}{q}-2$, for $\beta \in\left(1-\alpha, \frac{5 q-4}{3 q-4} \alpha-2\right]$ and for small enough $\epsilon>0$, we have

$$
\begin{aligned}
& 0 \leq 1+\beta-\alpha-s_{2} \leq \frac{4-q}{2 q} \beta, \\
& 0 \leq 2+\epsilon-2 \alpha-s_{2} \leq \frac{4-q}{2 q} \beta .
\end{aligned}
$$

From Lemma 4.1 and interpolation inequality we find

$$
\|\theta\|_{L_{t}^{\frac{2 q}{4-q}} \dot{H}^{1+\beta-\alpha-s_{2}}}+\|\theta\|_{L_{t}^{\frac{2 q}{4-q}} H^{2+\sigma+\epsilon-2 \alpha-s_{2}}} \lesssim 1+t .
$$


Let $q \in\left[2, \frac{20}{9}\right)$. If $\alpha \in\left(\frac{8 q-8}{7 q-4}, 1\right)$, we choose $s_{3}=2-2 \alpha<s_{2}$, then $\delta=\frac{2}{\alpha}\left(2-2 \alpha+\frac{q-2}{q}(2-\alpha)\right)$ and for $\beta \in\left(1-\alpha, \min \left\{2-2 \alpha, \frac{1-\alpha}{\frac{4}{\alpha}\left(1-\frac{1}{q}\right)-2}\right\}\right)$ we can get

$$
\begin{aligned}
& 0 \leq \beta-1+\alpha \leq \frac{4-q \delta}{2 q} \beta, \\
& 0 \leq \beta-1+\alpha \leq \frac{\beta}{2}
\end{aligned}
$$

Hence, we have

$$
\|\theta\|_{L_{t}^{\frac{2 q}{4-q \delta} \dot{H}^{1+\beta-\alpha-s_{3}}}}+\|\theta\|_{L_{t}^{2} \dot{H}^{1+\beta-\alpha-s_{3}}} \lesssim 1+t .
$$

The range of $\alpha$ and $\beta$ will monotonously shrink when $q$ increase. Hence for some $q \in\left[2, \frac{20}{9}\right)$ and for every $\widetilde{q} \in[2, q]$ we have for every $t \in \mathbb{R}^{+}$

$$
\|G(t)\|_{L^{\widetilde{q}}}^{\widetilde{q}}+\int_{0}^{t}\|G(\tau)\|_{L^{\frac{2 \widetilde{q}}{2-\alpha}}}^{\widetilde{\widetilde{a}}} d \tau \leq B(t) .
$$

\subsection{Estimation of $\|\omega\|_{L_{t}^{1} L^{\tilde{q}}}$ for every $\widetilde{q} \in[2, q]$ and for some $q \in\left[2, \frac{20}{9}\right)$}

In this subsection we give the estimate of $\|\omega\|_{L_{t}^{1} L^{\tilde{q}}}$ for $\widetilde{q} \in[2, q]$ for some $q \in\left[2, \frac{20}{9}\right)$.

Theorem 4.6. Consider (1.1) with $\sigma=0$ and $\gamma \geq 0$. Assume that $\left(\omega_{0}, \theta_{0}\right)$ satisfies the conditions in Theorem 1.1. Let $(\omega, \theta)$ be the corresponding solution of (1.1). For some $q \in\left[2, \frac{20}{9}\right)$ and for all $\widetilde{q} \in[2, q]$, when $(\alpha, \beta)$ satisfies the same conditions as in Theorem 4.5, we have

$$
\|\omega\|_{L_{t}^{1} L^{\tilde{q}}} \leq B(t) .
$$

Proof. we choose $q$ as in Theorem 4.5. Since $\beta>1-\alpha$, there exists a fixed constant $\rho>1$ such that $\frac{\beta}{\rho}>1-\alpha$. From the explicit formula of $G$ we have for every $\widetilde{q} \in[2, q]$

$$
\begin{aligned}
\|\omega\|_{L_{t}^{1} L^{\tilde{q}}} & \leq\|G\|_{L_{t}^{1} L^{\tilde{q}}}+\left\|\mathcal{R}_{\alpha} \theta\right\|_{L_{t}^{1} B_{\tilde{q}, 1}^{0}} \\
& \leq B(t)+t^{1-\frac{1}{\rho}}\left\|\mathcal{R}_{\alpha} \theta\right\|_{\widetilde{L}_{t}^{\rho} B_{\tilde{q}, 1}^{0}} .
\end{aligned}
$$

By a high-low frequency decomposition and a continuous embedding $B_{\tilde{q}, \infty}^{\frac{\beta}{\rho}} \hookrightarrow B_{\widetilde{q}, q}^{1-\alpha}$ we find

$$
\begin{aligned}
\left\|\mathcal{R}_{\alpha} \theta\right\|_{\widetilde{L}_{t}^{\rho} B_{\tilde{q}, 1}^{0}} & \leq\left\|\Delta_{-1} \mathcal{R}_{\alpha} \theta\right\|_{\widetilde{L}_{t}^{\rho} B_{\tilde{q}, 1}^{0}}+\left\|\left(\operatorname{Id}-\Delta_{-1}\right) \theta\right\|_{\widetilde{L}_{t}^{\rho} B_{\tilde{q}, 1}^{1-\alpha}} \\
& \lesssim\left\|\Delta_{-1} \theta\right\|_{L_{t}^{\rho} L^{\tilde{q}}}+\left\|\left(\mathrm{Id}-\Delta_{-1}\right) \theta\right\|_{\widetilde{L}_{t}^{\rho} B_{\tilde{q}, \infty}^{\frac{\beta}{\rho}}} \\
& \lesssim t^{\frac{1}{\rho}}\left\|\theta_{0}\right\|_{L^{\tilde{q}}}+\sup _{j \in \mathbb{N}} 2^{j \frac{\beta}{\rho}}\left\|\Delta_{j} \theta\right\|_{L_{t}^{\rho} L^{\tilde{q}}}
\end{aligned}
$$

Inserting the above estimate into the previous one and applying Lemma 2.4 we obtain

$$
\|\omega\|_{L_{t}^{1} L^{\tilde{q}}} \leq B(t)+C t^{1-\frac{1}{\rho}}\|\omega\|_{L_{t}^{1} L^{\tilde{q}}},
$$


where $C$ is an absolute constant depending only on $\widetilde{q}, \rho$ and $\left\|\theta_{0}\right\|_{L^{2} \cap L^{\infty}}$. If $C t^{1-\frac{1}{\rho}}=\frac{1}{2}$ equivalently, $t=\left(\frac{1}{2 C}\right)^{\frac{\rho}{\rho-1}}:=T_{0}$, then for every $t \leq T_{0}$

$$
\|\omega\|_{L_{t}^{1} L^{\tilde{q}}} \leq B(t) .
$$

Furthermore, if we evolve the system from the initial data $\left(u\left(T_{0}\right), \theta\left(T_{0}\right)\right)$, then using the time translation invariance and the fact that $\left\|\theta\left(T_{0}\right)\right\|_{L^{\widetilde{q}}} \leq$ $\left\|\theta_{0}\right\|_{L^{\tilde{q}}}$, we have for every $t \leq T_{0}$

$$
\|\omega\|_{L_{\left[T_{0}, T_{0}+t\right]}^{1} L \tilde{q} \leq B\left(T_{0}+t\right) .}
$$

Iterating like this, we finally get for every $t \in \mathbb{R}^{+}$

$$
\|\omega\|_{L_{t}^{1} L^{\widetilde{q}}} \leq B(t) .
$$

\subsection{Estimation of $\|G\|_{L_{t}^{1} B_{q, 1}^{s}}$}

In this subsection, we give the estimation of $\|G\|_{L_{t}^{1} B_{q, 1}^{s}}$. First we give a Lemma which is proved in [18]

Lemma 4.7. Let $p \in[2, \infty)$ and $\alpha \in[0,1]$. Then there exist two positive constants $c_{p}$ and $C_{p}$ such that for any $f \in \mathcal{S}^{\prime}$ and $j \in \mathbb{Z}$, we have

$$
c_{p} 2^{\frac{2 \alpha j}{p}}\left\|\Delta_{j} f\right\|_{L^{p}} \leq\left\|\Lambda^{\alpha}\left(\left|\Delta_{j} f\right|^{\frac{p}{2}}\right)\right\|_{L^{2}}^{\frac{2}{p}} \leq C_{p} 2^{\frac{2 \alpha j}{p}}\left\|\Delta_{j} f\right\|_{L^{p}} .
$$

Theorem 4.8. Consider (1.1) with $\sigma=0$ and $\gamma \geq 0$. Assume that $\left(\omega_{0}, \theta_{0}\right)$ satisfies the conditions in Theorem 1.1. Let $(\omega, \theta)$ be the corresponding solution of (1.1). Let $G$ defined as in (4.1). For $\alpha \in\left[\frac{19}{20}, 1\right), \beta \in\left(1-\alpha, \min \left\{2-2 \alpha, \frac{8}{3} \alpha-\right.\right.$ $\left.\left.2, \frac{5 \alpha(1-\alpha)}{11-10 \alpha}\right\}\right)$ and $\frac{9}{10} \leq s<2 \alpha-1$. We have

$$
\|G\|_{L_{t}^{1} B_{q, 1}^{s}} \leq B(t)
$$

where $q=\frac{20}{9}-\epsilon_{1}$ for $\epsilon_{1}>0$ is arbitrary small. In particular,

$$
\|G\|_{L_{t}^{1} B_{\infty, 1}^{0}} \leq B(t) .
$$

Proof. Applying the frequency localization operator $\Delta_{j}$ to the Eq. (4.1) we get

$$
\begin{aligned}
\partial_{t} \Delta_{j} G+u \cdot \nabla \Delta_{j} G+\Lambda^{\alpha} \Delta_{j} G= & -\left[\Delta_{j}, u \cdot \nabla\right] G-\Delta_{j}\left(\left[\mathcal{R}_{\alpha}, u \cdot \nabla\right] \theta\right) \\
& +\Delta_{j} \Lambda^{\beta-\alpha} \partial_{1} \theta \\
= & f_{j}(t) .
\end{aligned}
$$

Multiplying the above equation by $\left|\Delta_{j} G\right|^{q-2} \Delta_{j} G$ and integrating in the spatial variable we obtain

$$
\frac{1}{q} \frac{d}{d t}\left\|\Delta_{j} G(t)\right\|_{L^{q}}^{q}+\int_{\mathbb{R}^{2}} \Delta_{j} G\left|\Delta_{j} G\right|^{q-2} \Lambda^{\alpha} \Delta_{j} G d x=\int_{\mathbb{R}^{2}} f_{j} \Delta_{j} G\left|\Delta_{j} G\right|^{q-2} .
$$

Using Lemma 4.7 and Lemma 4.3, we can obtain

$$
\int_{\mathbb{R}^{2}} \Lambda^{\alpha} \Delta_{j} G\left|\Delta_{j} G\right|^{q-2} d x \geq c 2^{j \alpha}\left\|\Delta_{j} G\right\|_{L^{q}}^{q}
$$


with some positive constant $c$ independent of $j$. So we can obtain

$$
\frac{1}{q} \frac{d}{d t}\left\|\Delta_{j} G(t)\right\|_{L^{q}}^{q}+c 2^{j \alpha}\left\|\Delta_{j} G(t)\right\|_{L^{q}}^{q} \leq\left\|f_{j}\right\|_{L^{q}}\left\|\Delta_{j} G\right\|_{L^{q}}^{q-1} .
$$

Furthermore, we have

$$
\frac{d}{d t}\left\|\Delta_{j} G(t)\right\|_{L^{q}}+c 2^{j \alpha}\left\|\Delta_{j} G(t)\right\|_{L^{q}} \leq\left\|f_{j}\right\|_{L^{q}},
$$

then

$$
\begin{aligned}
\left\|\Delta_{j} G\right\|_{L_{t}^{1} L^{q}} \lesssim & 2^{-j \alpha}\left\|\Delta_{j} G(0)\right\|_{L^{q}}+2^{j(1+\beta-2 \alpha)}\left\|\Delta_{j} \theta\right\|_{L_{t}^{1} L^{q}} \\
& +2^{-j \alpha} \int_{0}^{t}\left\|\Delta_{j}\left(\left[\mathcal{R}_{\alpha}, u \cdot \nabla\right] \theta\right)\right\|_{L^{q}} d \tau \\
& +2^{-j \alpha} \int_{0}^{t}\left\|\left[\Delta_{j}, u \cdot \nabla\right] G\right\|_{L^{q}} d \tau .
\end{aligned}
$$

Now we deal with the second term on the right hand side of the above inequality. For every $j \in \mathbb{N}$, by Lemma 2.4 we have

$$
\left\|\Delta_{j} \theta\right\|_{L_{t}^{1} L^{q}} \leq 2^{-j \beta} B(t) .
$$

For the third term on the right hand side of the inequality (4.6). Using Theorem 3.3 we have

$$
\begin{aligned}
2^{-j \alpha} & \int_{0}^{t}\left\|\Delta_{j}\left(\left[\mathcal{R}_{\alpha}, u \cdot \nabla\right] \theta\right)\right\|_{L^{q}} d \tau \\
& \lesssim 2^{j(1+\epsilon-2 \alpha)} \int_{0}^{t}\left\|\left[\mathcal{R}_{\alpha}, u \cdot \nabla\right] \theta\right\|_{B_{q, \infty}^{\alpha-1}-\epsilon} d \tau \\
& \lesssim 2^{j(1+\epsilon-2 \alpha)} \int_{0}^{t}\|u\|_{\dot{B}_{q}^{1-\epsilon}}\left(\|\theta\|_{B_{\infty, \infty}^{0}}+\|\theta\|_{L^{\infty}}\right) d \tau \\
& \lesssim 2^{j(1+\epsilon-2 \alpha)}\|\omega\|_{L_{t}^{1} L^{q}}\left\|\theta_{0}\right\|_{L^{\infty}} \\
& \lesssim 2^{j(1+\epsilon-2 \alpha)} B(t) .
\end{aligned}
$$

For the fourth term on the right hand side of the inequality (4.6). Using Lemma 6.1 in the appendix, we have

$$
\begin{aligned}
2^{-j \alpha} & \int_{0}^{t}\left\|\left[\Delta_{j}, u \cdot \nabla\right] G\right\|_{L^{q}} d \tau \\
& \lesssim 2^{j(1+\epsilon-2 \alpha)} \int_{0}^{t} 2^{j(\alpha-1-\epsilon)}\left\|\left[\Delta_{j}, u \cdot \nabla\right] G\right\|_{L^{q}} d \tau \\
& \lesssim 2^{j(1+\epsilon-2 \alpha)} \int_{0}^{t}\left(\|G\|_{B_{q, \infty}^{\alpha-1}}+\|G\|_{L^{2}}+\left\|\theta_{0}\right\|_{L^{p} \cap L^{2}}\right)\|G\|_{B_{\infty}^{0}, \infty} d \tau \\
& \lesssim 2^{j(1+\epsilon-2 \alpha)} \int_{0}^{t}\left(\|G\|_{L^{q}}+\|G\|_{L^{2}}+\left\|\theta_{0}\right\|_{L^{p} \cap L^{2}}\right)\|G\|_{B_{q, 1}^{s}} d \tau \\
& \lesssim 2^{j(1+\epsilon-2 \alpha)} \int_{0}^{t} B(\tau)\|G\|_{B_{q, 1}^{s}} d \tau
\end{aligned}
$$


where $s \geq \frac{2}{q}$. Let $q \in \mathbb{N}$ be a number chosen later, then we have

$$
\begin{aligned}
\|G\|_{L_{t}^{1} B_{q, 1}^{s}} & \sum_{m<q} 2^{m s}\left\|\Delta_{m} G\right\|_{L_{t}^{1} L^{q}}+\sum_{m \geq q} 2^{m s}\left\|\Delta_{m} G\right\|_{L_{t}^{1} L^{q}} \\
\lesssim & 2^{q s} B(t)+\sum_{m \geq q} 2^{m s}\left\{2^{-m \alpha}\left\|\Delta_{m} G(0)\right\|_{L^{q}}+2^{m(1-2 \alpha)} B(t)\right. \\
& \left.\quad+2^{m(1+\epsilon-2 \alpha)} B(t)+2^{m(1+\epsilon-2 \alpha)} B(t)\|G\|_{L_{t}^{1} B_{q, 1}^{s}}\right\} \\
\lesssim & 2^{q s} B(t)+\sum_{m \geq q} 2^{m(s-\alpha)}\left\|\Delta_{m} G(0)\right\|_{L^{q}}+\sum_{m \geq q} 2^{m(s+1-2 \alpha)} B(t) \\
& +\sum_{m \geq q} 2^{m(s+1+\epsilon-2 \alpha)} B(t)+\sum_{m \geq q} 2^{m(s+1+\epsilon-2 \alpha)} B(t)\|G\|_{L_{t}^{1} B_{q, 1}^{s}} .
\end{aligned}
$$

If $\alpha>\frac{2+q}{2 q}$ and $\frac{2}{q} \leq s<2 \alpha-1$ we can take $\epsilon>0$ so small in the above statements such that $s<\alpha$ and $s+1+\epsilon-2 \alpha<0$. From Theorem 4.5, we know that for $2 \leq q<\frac{20}{9}$

$$
\max \left\{\frac{9 q-12}{8 q-8}, \frac{2+q}{2 q}\right\}<\alpha<1 .
$$

Through simple calculations, we easily know that the range of $\alpha$ can be the largest one when we choose $q=\frac{20}{9}-\epsilon_{1}$ for $\epsilon_{1}>0$ is arbitrary small. So, we have

$$
\begin{aligned}
& \alpha \in\left[\frac{19}{20}, 1\right) \\
& \beta \in\left(1-\alpha, \min \left\{2-2 \alpha, \frac{8}{3} \alpha-2, \frac{5 \alpha(1-\alpha)}{11-10 \alpha}\right\}\right) \\
& \frac{9}{10} \leq s<2 \alpha-1 .
\end{aligned}
$$

Hence, we finally obtain

$$
\|G\|_{L_{t}^{1} B_{q, 1}^{s}} \leq B(t) 2^{q s}+2^{-q(2 \alpha-s-s-\epsilon)} B(t)\|G\|_{L_{t}^{1} B_{q, 1}^{s}} .
$$

Choosing $q$ such that $2^{-q(2 \alpha-s-1-\epsilon)} B(t) \approx \frac{1}{2}$. Thus we obtain for every $t \in \mathbb{R}^{+}$

$$
\|G\|_{L_{t}^{1} B_{q, 1}^{s}} \leq B(t)
$$

By embedding this immediately leads to

$$
\|G\|_{L_{t}^{1} B_{\infty, 1}^{s-\frac{9}{10}}} \leq B(t) .
$$




\subsection{Estimation of $\|\omega\|_{L_{t}^{1} B_{\infty, 1}^{0, \gamma}}$ and $\|\theta\|_{L_{t}^{1} B_{\infty, 1}^{0, \gamma}}$}

Lemma 4.9. Consider (1.1) with $\sigma=0$ and $\gamma \geq 0$. Assume that $\left(\omega_{0}, \theta_{0}\right)$ satisfies the conditions in Theorem 1.1. Let $(\omega, \theta)$ be the corresponding solution of (1.1). Take $p$ large enough such that $\frac{2}{p}+1<\alpha+\beta$. Then we have

$$
\|\omega\|_{L_{t}^{1} L^{p}} \leq B(t) .
$$

Proof. For $\alpha+\beta>1$, we choose $\rho>1$ such that $\frac{\beta}{\rho}>1-\alpha$ From the definition of $G$ as in (4.1), we have

$$
\begin{aligned}
\|\omega\|_{L_{t}^{1} L^{p}} & \leq\|G\|_{L_{t}^{1}\left(B_{\infty, 1}^{0} \cap L^{2}\right)}+\left\|\mathcal{R}_{\alpha} \theta\right\|_{L_{t}^{1} B_{p, 1}^{0}} \\
& \leq B(t)+t^{1-\frac{1}{\rho}}\left\|\mathcal{R}_{\alpha} \theta\right\|_{\widetilde{L}_{t}^{\rho} B_{p, 1}^{0}} .
\end{aligned}
$$

Then through the same idea in the proof of Theorem 4.6, we can easily get the conclusion.

Now we state the main theorem in this section.

Theorem 4.10. Consider (1.1) with $\sigma=0, \gamma \geq 0$ and $(\alpha, \beta)$ satisfies conditions as in Theorem 4.8. Assume that $\left(\omega_{0}, \theta_{0}\right)$ satisfies the conditions in Theorem 1.1. Let $(\omega, \theta)$ be the corresponding solution of (1.1). Then we have

$$
\begin{aligned}
& \|\omega\|_{L_{t}^{1} B_{\infty, 1}^{0, \gamma}} \leq B(t) \\
& \|\theta\|_{L_{t}^{1} B_{\infty, 1}^{0, \gamma}} \leq B(t) .
\end{aligned}
$$

Proof. Since for $s>\frac{2}{q}$ where $q$ as in Theorem 4.8, we have

$$
\begin{aligned}
\|G\|_{B_{\infty, 1}^{0, \gamma}} & =\sum_{j \geq-1}(1+|j|)^{\gamma}\left\|\Delta_{j} G\right\|_{L^{\infty}} \\
& \leq \sum_{j \geq-1}(1+|j|)^{\gamma} 2^{\frac{2}{q} j} 2^{-j s} 2^{j s}\left\|\Delta_{j} G\right\|_{L^{q}} \\
& \leq C\|G\|_{B_{q, 1}^{s}} .
\end{aligned}
$$

From Theorem 4.8, we obtain

$$
\|G\|_{L_{t}^{1} B_{\infty, 1}^{0, \gamma}} \leq C\|G\|_{L_{t}^{1} B_{q, 1}^{s}} \leq B(t)
$$

Using the definition of $G$ as in (4.1), we have

$$
\begin{aligned}
\|\omega\|_{L_{t}^{1} B_{\infty, 1}^{0, \gamma}} & \leq\|G\|_{L_{t}^{1} B_{\infty, 1}^{0, \gamma}}+\left\|\mathcal{R}_{\alpha} \theta\right\|_{L_{t}^{1} B_{\infty, 1}^{0, \gamma}} \\
& \leq B(t)+\left\|\mathcal{R}_{\alpha} \theta\right\|_{L_{t}^{1} B_{\infty, 1}^{0, \gamma}}
\end{aligned}
$$


For the second term, we have

$$
\begin{aligned}
& \left\|\mathcal{R}_{\alpha} \theta\right\|_{L_{t}^{1} B_{\infty, 1}^{0, \gamma}} \\
& \quad=\sum_{j \geq-1}(1+|j|)^{\gamma}\left\|\Delta_{j} \mathcal{R}_{\alpha} \theta\right\|_{L_{t}^{1} L^{\infty}} \\
& \quad \lesssim\left\|\Delta_{-1} \mathcal{R}_{\alpha} \theta\right\|_{L_{t}^{1} L^{\infty}}+\sum_{j \geq 0}(1+|j|)^{\gamma}\left\|\Delta_{j} \mathcal{R}_{\alpha} \theta\right\|_{L_{t}^{1} L^{\infty}} \\
& \quad \lesssim\left\|\Delta_{-1} \theta\right\|_{L_{t}^{1} L^{\infty}}+\sum_{j=0}^{\infty} 2^{j(1-\alpha)}(1+|j|)^{\gamma}\left\|\Delta_{j} \theta\right\|_{L_{t}^{1} L^{\infty}} \\
& \quad \lesssim t\left\|\theta_{0}\right\|_{L^{2}}+\sum_{j=0}^{\infty} 2^{-j\left(\beta+\alpha-1-\frac{2}{p}\right)}(1+|j|)^{\gamma} 2^{j \beta}\left\|\Delta_{j} \theta\right\|_{L_{t}^{1} L^{p}} \\
& \quad \lesssim t\left\|\theta_{0}\right\|_{L^{2}}+\left\|\theta_{0}\right\|_{L^{p}}+\left\|\theta_{0}\right\|_{L^{\infty}}\|\omega\|_{L_{t}^{1} L^{p}} \\
& \quad \lesssim B(t),
\end{aligned}
$$

where $\frac{2}{p}+1<\alpha+\beta$ and we have used Lemma 2.4 and the estimation of $\|\omega\|_{L_{t}^{1} L^{p}}$. Hence we obtain

$$
\|\omega\|_{L_{t}^{1} B_{\infty, 1}^{0, \gamma}} \leq B(t)
$$

For $\theta$ we have

$$
\begin{aligned}
\|\theta\|_{L_{t}^{1} B_{\infty, 1}^{0, \gamma}} & =\sum_{j=-1}^{\infty}(1+|j|)^{\gamma}\left\|\Delta_{j} \theta\right\|_{L_{t}^{1} L^{\infty}} \\
& \lesssim t\left\|\theta_{0}\right\|_{L^{2}}+\sum_{j=0}^{\infty}(1+|j|)^{\gamma} 2^{j \frac{2}{p}} 2^{-j \beta} 2^{j \beta}\left\|\Delta_{j} \theta\right\|_{L_{t}^{1} L^{p}} \\
& \lesssim t\left\|\theta_{0}\right\|_{L^{2}}+\left\|\theta_{0}\right\|_{L^{p}}+\left\|\theta_{0}\right\|_{L^{\infty}}\|\omega\|_{L_{t}^{1} L^{p}} \\
& \lesssim B(t),
\end{aligned}
$$

where $\frac{2}{p}+1<\alpha+\beta$. Thus, the proof is complete.

\subsection{Estimation of $\|\theta\|_{\widetilde{L}_{t}^{\infty}\left(H^{1-\alpha} \cap B_{\infty, 1}^{1-\alpha+\epsilon}\right)},\|\omega\|_{L_{t}^{\infty} L^{p}}$.}

The following is the main result of this subsection.

Theorem 4.11. Consider (1.1) with $\sigma=0, \gamma \geq 0$ and $(\alpha, \beta)$ satisfies conditions as in Theorem 4.8. Assume that $\left(\omega_{0}, \theta_{0}\right)$ satisfies the conditions in Theorem 1.1. Let $(\omega, \theta)$ be the corresponding solution of (1.1). Then for arbitrary small $\epsilon>0$ and any $p \geq 2$ we have

$$
\begin{aligned}
& \|\theta\|_{\widetilde{L}_{t}^{\infty}\left(H^{1-\alpha} \cap B_{\infty, 1}^{1-\alpha+\epsilon}\right)}+\|\theta\|_{\widetilde{L}_{t}^{1}\left(H^{1-\alpha+\beta} \cap B_{\infty, 1}^{1-\alpha+\beta+\epsilon}\right)} \leq B(t) \\
& \|\omega\|_{L_{t}^{\infty} L^{p}} \leq B(t) .
\end{aligned}
$$

Proof. Our proof can be divided into three steps. Step 1: let us give the estimation of $\|u\|_{L_{t}^{1} B_{\infty, 1}^{1}}$. Since for $s>\frac{2}{q}$ where $q$ as in Theorem 4.8, take $\epsilon>0$ 
such that $s-\frac{2}{q}-\epsilon>0$, we have

$$
\begin{aligned}
\|G\|_{B_{\infty}^{\epsilon}, 1} & =\sum_{j \geq-1} 2^{j \epsilon}\left\|\Delta_{j} G\right\|_{L^{\infty}} \\
& \leq \sum_{j \geq-1} 2^{j \epsilon} 2^{j \frac{2}{q}} 2^{-j s} 2^{j s}\left\|\Delta_{j} G\right\|_{L^{q}} \\
& \leq \sum_{j \geq-1} 2^{-j\left(s-\frac{2}{q}-\epsilon\right)} 2^{j s}\left\|\Delta_{j} G\right\|_{L^{q}} \\
& \leq C\|G\|_{B_{q, 1}^{s}} .
\end{aligned}
$$

From Theorem 4.8, we obtain $\|G\|_{L_{t}^{1} B_{\infty, 1}^{\epsilon}} \lesssim\|G\|_{L_{t}^{1} B_{q, 1}^{s}} \lesssim B(t)$. Using the definition of $G$ as in (4.1), we have

$$
\|\omega\|_{L_{t}^{1} B_{\infty, 1}^{\epsilon}} \leq\|G\|_{L_{t}^{1} B_{\infty, 1}^{\epsilon}}+\left\|\mathcal{R}_{\alpha} \theta\right\|_{L_{t}^{1} B_{\infty, 1}^{\epsilon}} \leq B(t)+\left\|\mathcal{R}_{\alpha} \theta\right\|_{L_{t}^{1} B_{\infty, 1}^{\epsilon}}
$$

For the second term, we have

$$
\begin{aligned}
\left\|\mathcal{R}_{\alpha} \theta\right\|_{L_{t}^{1} B_{\infty, 1}^{\epsilon}} & =\sum_{j \geq-1} 2^{j \epsilon}\left\|\Delta_{j} \mathcal{R}_{\alpha} \theta\right\|_{L_{t}^{1} L^{\infty}} \\
& \lesssim\left\|\Delta_{-1} \mathcal{R}_{\alpha} \theta\right\|_{L_{t}^{1} L^{\infty}}+\sum_{j \geq 0} 2^{j \epsilon}\left\|\Delta_{j} \mathcal{R}_{\alpha} \theta\right\|_{L_{t}^{1} L^{\infty}} \\
& \lesssim\left\|\Delta_{-1} \theta\right\|_{L_{t}^{1} L^{\infty}}+\sum_{j=0}^{\infty} 2^{j(1-\alpha)} 2^{j \epsilon}\left\|\Delta_{j} \theta\right\|_{L_{t}^{1} L^{\infty}} \\
& \lesssim t\left\|\theta_{0}\right\|_{L^{2}}+\sum_{j=0}^{\infty} 2^{-j\left(\beta+\alpha-1-\frac{2}{p}\right)} 2^{j \epsilon} 2^{j \beta}\left\|\Delta_{j} \theta\right\|_{L_{t}^{1} L^{p}} \\
& \lesssim t\left\|\theta_{0}\right\|_{L^{2}}+\left\|\theta_{0}\right\|_{L^{p}}+\left\|\theta_{0}\right\|_{L^{\infty}}\|\omega\|_{L_{t}^{1} L^{p}} \\
& \lesssim B(t) .
\end{aligned}
$$

where $\frac{2}{p}+1+\epsilon<\alpha+\beta$ and we have used Lemma 2.4 and the estimation of $\|\omega\|_{L_{t}^{1} L^{p}}$. Hence, we obtain $\|\omega\|_{L_{t}^{1} B_{\infty, 1}^{\epsilon}} \leq B(t)$. On the other hand, by HardyLittlewood-Sobolev inequality, we obtain

$$
\left\|\Delta_{-1} u\right\|_{L_{t}^{1} L^{\infty}} \lesssim\left\|\Delta_{-1} \Lambda^{\epsilon-1} \omega\right\|_{L_{t}^{1} L^{\infty}} \lesssim\left\|\Delta_{-1} \Lambda^{\epsilon-1} \omega\right\|_{L_{t}^{1} L^{\frac{2}{\epsilon}}} \lesssim\|\omega\|_{L_{t}^{1} L^{2}} \lesssim B(t)
$$

From the above statements, we finally get

$$
\begin{aligned}
\|u\|_{L_{t}^{1} B_{\infty, 1}^{1}} & \lesssim\left\|\Delta_{-1} u\right\|_{L_{t}^{1} L^{\infty}}+\sum_{q \in \mathbb{N}}\left\|\Delta_{q} \nabla u\right\|_{L_{t}^{1} L^{\infty}} \\
& \lesssim\left\|\Delta_{-1} u\right\|_{L_{t}^{1} L^{\infty}}+\|\omega\|_{L_{t}^{1} B_{\infty, 1}^{\epsilon}} \\
& \lesssim B(t) .
\end{aligned}
$$


Step 2: estimation of $\theta$. Using Lemma 2.5 and the result in step 1 , we can obtain

$$
\begin{aligned}
& \|\theta\|_{\widetilde{L}_{t}^{\infty}\left(H^{1-\alpha} \cap B_{\infty, 1}^{1-\alpha+\epsilon}\right)}+\|\theta\|_{\widetilde{L}_{t}^{1}\left(H^{1-\alpha+\beta} \cap B_{\infty, 1}^{1-\alpha+\beta+\epsilon}\right)} \\
& \quad \lesssim\|\theta\|_{\widetilde{L}_{t}^{\infty}\left(H^{1-\alpha} \cap B_{\infty, 1}^{1-\alpha+\epsilon}\right)}+\left\|\left(\mathrm{Id}-\Delta_{-1}\right) \theta\right\|_{\widetilde{L}_{t}^{1}\left(H^{1-\alpha+\beta} \cap B_{\infty, 1}^{1-\alpha+\beta+\epsilon}\right)} \\
& \quad+t\left\|\theta^{0}\right\|_{L^{2} \cap L^{\infty}} \\
& \quad \lesssim e^{C\|u\|_{L_{t}^{1} B_{\infty, 1}^{1}}} \\
& \quad \lesssim B(t) .
\end{aligned}
$$

Step 3: estimation of $\omega$. By the Eq. (4.1) and Lemma 2.3, we have

$$
\begin{aligned}
\|G(t)\|_{L^{p}} \leq & \left\|G_{0}\right\|_{L^{p}}+\int_{0}^{t}\left\|\left[\mathcal{R}_{\alpha}, u \cdot \nabla\right] \theta(\tau)\right\|_{L^{p}} d \tau \\
& +\int_{0}^{t}\left\|\Lambda^{\beta} \mathcal{R}_{\alpha} \theta(\tau)\right\|_{L^{p}} d \tau .
\end{aligned}
$$

For the first integral of the RHS, using Theorem 3.3 with $s=0$ yields

$$
\begin{aligned}
\left\|\left[\mathcal{R}_{\alpha}, u \cdot \nabla\right] \theta(\tau)\right\|_{L^{p}} & \leq\left\|\left[\mathcal{R}_{\alpha}, u \cdot \nabla\right] \theta(\tau)\right\|_{B_{p, 1}^{0}} \\
& \lesssim\|u(\tau)\|_{\dot{B}_{p, \infty}^{1-\epsilon}}\left(\|\theta(\tau)\|_{B_{\infty, 1}^{1-\alpha+\epsilon}}+\|\theta(\tau)\|_{L^{\infty}}\right) \\
& \lesssim B(\tau)\|\omega(\tau)\|_{L^{p}}
\end{aligned}
$$

For the second integral of the RHS, we have

$$
\begin{aligned}
& \int_{0}^{1}\left\|\Lambda^{\beta} \mathcal{R}_{\alpha} \theta(\tau)\right\|_{L^{p}} d \tau \\
& \quad \lesssim\left\|\Delta_{-1} \theta\right\|_{L_{t}^{1} L^{p}}+\left\|\left(\mathrm{Id}-\Delta_{-1}\right) \theta\right\|_{L_{t}^{1} B_{p, 1}^{1-\alpha+\beta}} \\
& \quad \lesssim\|\theta\|_{L_{t}^{1} L^{p}}+e^{C\|\nabla u\|_{L_{t}^{1} L^{\infty}}}\left\|\theta_{0}\right\|_{B_{p, 1}^{1-\alpha}} \\
& \quad \lesssim B(t)
\end{aligned}
$$

Hence, gathering the upper estimates we obtain

$$
\begin{aligned}
\|\omega(t)\|_{L^{p}} & \leq\|G(t)\|_{L^{p}}+\left\|\mathcal{R}_{\alpha} \theta(t)\right\|_{L^{p}} \\
& \leq B(t)+\int_{0}^{t} B(\tau)\|\omega(\tau)\|_{L^{p}} d \tau .
\end{aligned}
$$

Gronwall's inequality yields

$$
\|\omega(t)\|_{L^{p}} \leq B(t) .
$$

At this stage, we can construct approximation system and use similar methods in [4] to prove the existence of the solution. 


\section{Uniqueness}

In this section, we prove the uniqueness. For convenience of the reader, we clarify some notations. Let $\left(w^{1}, \theta^{1}\right)$ and $\left(\omega^{2}, \theta^{2}\right)$ be two solutions of system (1.1) with $\sigma=0, \gamma \geq 0 . u^{1}$ and $u^{2}$ be the corresponding velocity fields, namely

$$
u^{j}=\nabla^{\perp} \psi^{j}, \quad \Delta \psi^{j}=(\log (\operatorname{Id}-\Delta))^{\gamma} \omega^{j}, \quad j=1,2 .
$$

Let $v^{j}=(\log (\operatorname{Id}-\Delta))^{-\gamma} u^{j}, \quad j=1,2$. Denote

$$
u=u^{2}-u^{1}, \quad \theta=\theta^{2}-\theta^{1}, \quad v=v^{2}-v^{1}, \quad p=p^{2}-p^{1} .
$$

Then we give two crucial estimates

Lemma 5.1. Assume that $\theta$ satisfies

$$
\partial_{t} \theta+u \cdot \nabla \theta^{1}+u^{2} \cdot \nabla \theta+\Lambda^{\beta} \theta=0, \quad 0 \leq \beta \leq 2 .
$$

Then, for any $t>0$,

$$
\begin{aligned}
\|\theta(t)\|_{B_{2, \infty}^{-1}} \leq & \|\theta(0)\|_{B_{2, \infty}^{-1}}+C \int_{0}^{t}\|v(s)\|_{L^{2}}\left\|\theta^{1}\right\|_{B_{\infty, 1}^{1-\alpha, \gamma}} d s \\
& +C \int_{0}^{1}\left\|\omega^{2}(s)\right\|_{B_{\infty, 1}^{0, \gamma}}\|\theta(s)\|_{B_{2, \infty}^{-\alpha}} d s .
\end{aligned}
$$

Proof. Let $j \geq-1$. Applying $\Delta_{j}$ to (5.1), taking the inner product of $\Delta_{j} \theta$ with the resulting equation and applying Hölder's inequality, we obtain

$$
\frac{d}{d t}\left\|\Delta_{j} \theta\right\|_{L^{2}} \leq\left\|\Delta_{j}\left(u \cdot \nabla \theta^{1}\right)\right\|_{L^{2}}+\left\|\Delta_{j}\left(u^{2} \cdot \nabla \theta\right)\right\|_{L^{2}} .
$$

To estimate the first term, we write

$$
\Delta_{j}\left(u \cdot \nabla \theta^{1}\right)=J_{1}+J_{2}+J_{3},
$$

where $J_{1}, J_{2}$ and $J_{3}$ are given by

$$
\begin{aligned}
& J_{1}=\sum_{|j-k| \leq 2} \Delta_{j}\left(S_{k-1} u \nabla \Delta_{k} \theta^{1}\right), \\
& J_{2}=\sum_{|j-k| \leq 2} \Delta_{j}\left(\Delta_{k} u \nabla S_{k-1} \theta^{1}\right), \\
& J_{3}=\sum_{k \geq j-1} \Delta_{j}\left(\Delta_{k} u \nabla \widetilde{\Delta}_{k} \theta^{1}\right) .
\end{aligned}
$$

$J_{1}, J_{2}$ and $J_{3}$ can be estimated as follows.

$$
\begin{aligned}
\left\|J_{1}\right\|_{L^{2}} & \leq C 2^{j}\left\|S_{j-1} u\right\|_{L^{2}}\left\|\Delta_{j} \theta^{1}\right\|_{L^{\infty}} \\
& \leq C 2^{\alpha j} 2^{(1-\alpha) j}\|v\|_{L^{2}}(1+|j|)^{\gamma}\left\|\Delta_{j} \theta^{1}\right\|_{L^{\infty}} \\
& \leq C 2^{j \alpha}\|v\|_{L^{2}}\left\|\theta^{2}\right\|_{B_{\infty, 1}^{1-\alpha, \gamma}}
\end{aligned}
$$




$$
\begin{aligned}
\left\|J_{2}\right\|_{L^{2}} & \leq C\left\|\Delta_{j} u\right\|_{L^{2}}\left\|S_{j-1} \nabla \theta\right\|_{L^{\infty}} \\
& \leq C\left\|\Delta_{j} v\right\|_{L^{2}}(1+|j|)^{\gamma} \sum_{m \leq j-2} 2^{m}\left\|\Delta_{m} \theta^{1}\right\|_{L^{\infty}} \\
& \leq C 2^{j \alpha}\|v\|_{B_{2, \infty}^{0}} \sum_{m \leq j-2} \frac{2^{m \alpha}(1+|m|)^{-\gamma}}{2^{j \alpha}(1+|j|)^{-\gamma}} 2^{(1-\alpha) m}(1+|m|)^{\gamma}\left\|\Delta_{m} \theta^{1}\right\|_{L^{\infty}} \\
& \leq C 2^{j \alpha}\|v\|_{B_{2, \infty}^{0}}\left\|\theta^{1}\right\|_{B_{\infty, 1}^{1-\alpha, \gamma}} \\
\left\|J_{3}\right\|_{L^{2}} & \leq C 2^{j} \sum_{k \geq j-1}(1+|k|)^{\gamma}\left\|\Delta_{k} \theta^{1}\right\|_{L^{\infty}}\left\|\Delta_{k} v\right\|_{L^{2}} \\
& \leq C 2^{j \alpha} 2^{j(1-\alpha)} \sum_{k \geq j-1} 2^{-k(1-\alpha)}(1+|k|)^{\gamma}\left\|\Delta_{k} \theta^{1}\right\|_{L^{\infty} 2^{k(1-\alpha)}\left\|\Delta_{k} v\right\|_{L^{2}}} \\
& \leq C 2^{j \alpha} \sum_{k \geq j-1} 2^{(j-k)(1-\alpha)}(1+|k|)^{\gamma}\left\|\Delta_{k} \theta^{1}\right\|_{L^{\infty}} 2^{k(1-\alpha)}\left\|\Delta_{k} v\right\|_{L^{2}} \\
& \leq C 2^{j \alpha}\|v\|_{B_{2, \infty}^{0}}\left\|\theta^{1}\right\|_{B_{\infty, 1}^{1-\alpha, \gamma}}
\end{aligned}
$$

To estimate the second term, we write

$$
\Delta_{j}\left(u^{2} \cdot \nabla \theta\right)=K_{1}+K_{2}+K_{3}+K_{4}+K_{5},
$$

where

$$
\begin{aligned}
K_{1} & =\sum_{|j-k| \leq 2}\left[\Delta_{j}, S_{k-1} u^{2} \cdot \nabla\right] \Delta_{k} \theta, \\
K_{2} & =\sum_{|j-k| \leq 2}\left(S_{k-1} u^{2}-S_{j} u^{2}\right) \cdot \nabla \Delta_{j} \Delta_{k} \theta, \\
K_{3} & =S_{j} u^{2} \cdot \nabla \Delta_{j} \theta \\
K_{4} & =\sum_{|j-k| \leq 2} \Delta_{j}\left(\Delta_{k} u^{2} \cdot \nabla S_{k-1} \theta\right), \\
K_{5} & =\sum_{k \geq j-1} \Delta_{j}\left(\Delta_{k} u^{2} \cdot \nabla \widetilde{\Delta}_{k} \theta\right) .
\end{aligned}
$$

Since $\nabla \cdot u^{2}=0$, we know that

$$
\int \Delta_{j} \theta K_{3} d x=0
$$

By a standard commutator estimate, we obtain

$$
\begin{aligned}
\left\|K_{1}\right\|_{L^{2}} & \leq C\left\|x \Phi_{j}(x)\right\|_{L^{1}}\left\|\nabla S_{j-1} u^{2}\right\|_{L^{\infty}}\left\|\nabla \Delta_{j} \theta\right\|_{L^{2}} \\
& \leq C\left\|x \Phi_{j}(x)\right\|_{L^{1}}\left\|\omega^{2}\right\|_{B_{\infty, 1}^{0, \gamma}}\left\|\Delta_{j} \theta\right\|_{L^{2}}
\end{aligned}
$$


where $\Phi_{j}(x)$ is the kernel of the operator $\Delta_{j}$. For $j \geq j_{0}$ with $j_{0}=2$, we apply Bernstein's inequality to obtain

$$
\begin{aligned}
\left\|K_{2}\right\|_{L^{2}} & \leq C\left\|\Delta_{j} u^{2}\right\|_{L^{\infty}}\left\|\nabla \Delta_{j} \theta\right\|_{L^{2}} \\
& \leq C\left\|\Delta_{j} \nabla u^{2}\right\|_{L^{\infty}}\left\|\Delta_{j} \theta\right\|_{L^{2}} \\
& \leq C\left\|\omega^{2}\right\|_{B_{\infty, 1}^{0, \gamma}}\left\|\Delta_{j} \theta\right\|_{L^{2}} .
\end{aligned}
$$

Again, for $j \geq j_{0}$ with $j_{0}=2$, we have

$$
\begin{aligned}
\left\|K_{4}\right\|_{L^{2}} \leq C\left\|\Delta_{j} u^{2}\right\|_{L^{\infty}}\left\|S_{j-1} \nabla \theta\right\|_{L^{2}} \\
\leq C 2^{j \alpha}\left\|\Delta_{j} \nabla u^{2}\right\|_{L^{\infty}} \sum_{m \leq j-2} 2^{(1+\alpha)(m-j)} 2^{-m \alpha}\left\|\Delta_{m} \theta\right\|_{L^{2}} \\
\leq C 2^{j \alpha}\left\|\omega^{2}\right\|_{B_{\infty, 1}^{0, \gamma},\|\theta\|_{B_{2, \infty}^{-\alpha}}} \\
\left\|K_{5}\right\|_{L^{2}} \leq C 2^{j} \sum_{k \geq j-1}\left\|\Delta_{k} u^{2}\right\|_{L^{\infty}}\left\|\Delta_{k} \theta\right\|_{L^{2}} \\
\leq C 2^{j \alpha} \sum_{k \geq j-1} 2^{-k} 2^{k(1-\alpha)}\left\|\Delta_{k} \nabla u^{2}\right\|_{L^{\infty}}\left\|_{\Delta_{k}} \theta\right\|_{L^{2}} \\
\leq C 2^{j \alpha} \sum_{k \geq j-1}\left\|\Delta_{k} \nabla u^{2}\right\|_{L^{\infty}} 2^{-k \alpha}\left\|\Delta_{k} \theta\right\|_{L^{2}} \\
\leq 2^{j \alpha}\left\|\omega^{2}\right\|_{B_{\infty, 1}^{0, \gamma}}\|\theta\|_{B_{2, \infty}^{-\alpha}} .
\end{aligned}
$$

From all the above estimates, we obtain

$$
\begin{aligned}
\frac{d}{d t}\left\|\Delta_{j} \theta\right\|_{L^{2}} \leq & C 2^{j \alpha}\|v\|_{L^{2}}\left\|\theta^{1}\right\|_{B_{\infty, 1}^{1-\alpha, \gamma}}+C\left\|\omega^{2}\right\|_{B_{\infty, 1}^{0, \gamma}}\left\|\Delta_{j} \theta\right\|_{L^{2}} \\
& +C 2^{j \alpha}\left\|\omega^{2}\right\|_{B_{\infty, 1}^{0, \gamma}}\|\theta\|_{B_{2, \infty}^{-\alpha}} .
\end{aligned}
$$

Integrating in time leads to

$$
\begin{aligned}
\left\|\Delta_{j} \theta(t)\right\|_{L^{2}} \leq & \left\|\Delta_{j} \theta(0)\right\|_{L^{2}}+C 2^{j \alpha} \int_{0}^{t}\|v(s)\|_{L^{2}}\left\|\theta^{1}(s)\right\|_{B_{\infty, 1}^{1-\alpha, \gamma}} d s \\
& +C 2^{j \alpha} \int_{0}^{t}\left\|\omega^{2}(s)\right\|_{B_{\infty, 1}^{0, \gamma},\|\theta(s)\|_{B_{2, \infty}^{-\alpha}} d s}
\end{aligned}
$$

Hence, we finally get

$$
\begin{aligned}
\|\theta(t)\|_{B_{2, \infty}^{-\alpha}} \leq & \|\theta(0)\|_{B_{2, \infty}^{-\alpha}}+C \int_{0}^{t}\|v(s)\|_{L^{2}}\left\|\theta^{1}(s)\right\|_{B_{\infty, 1}^{1-\alpha, \gamma}} d s \\
& +C \int_{0}^{t}\left\|\omega^{2}(s)\right\|_{B_{\infty, 1}^{0, \gamma}}\|\theta(s)\|_{B_{2, \infty}^{-\alpha}} d s .
\end{aligned}
$$

Lemma 5.2. Assume that $v$ satisfies

$$
\partial_{t} v+u^{2} \cdot \nabla v+u \cdot \nabla v^{1}-\sum_{j=1}^{2}\left(u_{j}^{2} \nabla v_{j}+u_{j} \nabla v_{j}^{1}\right)+\Lambda^{\alpha} v=-\nabla p+\theta e_{2},
$$


for $\alpha \in(0,1]$. Then

$$
\begin{aligned}
\|v(t)\|_{B_{2, \infty}^{0}} \leq & \|v(0)\|_{B_{2, \infty}^{0}}+\sup _{0 \leq s \leq t}\|\theta(s)\|_{B_{2, \infty}^{-\alpha}} \\
& +C \int_{0}^{t}\|v(s)\|_{L^{2}}\left(\left\|\omega^{1}(s)\right\|_{B_{\infty, 1}^{0, \gamma}}+\left\|\omega^{2}(s)\right\|_{B_{\infty, 1}^{0, \gamma}}\right) d s .
\end{aligned}
$$

Proof. Let $j \geq-1$. After applying $\Delta_{j}$ to equation (5.4), taking the inner product with $\Delta_{j} v$ and integrating by parts, we find

$$
\frac{1}{2} \frac{d}{d t}\left\|\Delta_{j} v\right\|_{L^{2}}^{2}+c 2^{j \alpha}\left\|\Delta_{j} v\right\|_{L^{2}}^{2}=L_{1}+L_{2}+L_{3}+L_{4}+L_{5},
$$

where

$$
\begin{aligned}
& L_{1}=-\int \Delta_{j} v \cdot \Delta_{j}\left(u^{2} \cdot \nabla v\right) d x \\
& L_{2}=-\int \Delta_{j} v \cdot \Delta_{j}\left(u \cdot \nabla v^{1}\right) d x \\
& L_{3}=-\sum_{n=1}^{2} \int \Delta_{j} v \cdot \Delta_{j}\left(u_{n}^{2} \nabla v_{n}\right) d x \\
& L_{4}=-\sum_{n=1}^{2} \int \Delta_{j} v \cdot \Delta_{j}\left(u_{n} \nabla v_{n}^{1}\right) d x \\
& L_{5}=-\int \Delta_{j} v_{2} \cdot \Delta_{j} \theta .
\end{aligned}
$$

To estimate $L_{1}$, we decompose $\Delta_{j}\left(u^{2} \cdot \nabla v\right)$ as in (5.3) and bound the components in a similar fashion as the above Lemma. We obtain after applying Hölder's inequality

$$
\left|L_{1}\right| \leq C\left\|\Delta_{j} v\right\|_{L^{2}}\|v\|_{L^{2}}\left\|\omega^{2}\right\|_{B_{\infty}^{0, \gamma}, 1} .
$$

To deal with $L_{2}$, similar to the proof in the above Lemma we obtain

$$
\left|L_{2}\right| \leq C\left\|\Delta_{j} v\right\|_{L^{2}}\|v\|_{L^{2}}\left\|\omega^{1}\right\|_{B_{\infty, 1}^{0, \gamma}} .
$$

To handle $L_{3}$, we integrate by part and use the divergence-free condition to obtain

$$
L_{3}=\sum_{n=1}^{2} \int \Delta_{j} v \cdot \Delta_{j}\left(v_{n} \nabla u_{n}^{2}\right) .
$$

Then using the same idea as in Lemma 5.1 we have

$$
\left|L_{3}\right| \leq C\left\|\Delta_{j} v\right\|_{L^{2}}\|v\|_{L^{2}}\left\|\omega^{2}\right\|_{B_{\infty}^{0, \gamma},} .
$$

We can easily notice that $L_{4}$ admits the same bound as $L_{2} . L_{5}$ can be bounded by applying Hölder's inequality

$$
\left|L_{5}\right| \leq\left\|\Delta_{j} v\right\|_{L^{2}}\left\|\Delta_{j} \theta\right\|_{L^{2}} \leq 2^{j \alpha}\left\|\Delta_{j} v\right\|_{L^{2}}\|\theta\|_{B_{2, \infty}^{-\alpha}} .
$$


From all the above statements, we find

$$
\begin{aligned}
& \frac{d}{d t}\left\|\Delta_{j} v\right\|_{L^{2}}+2^{j \alpha}\left\|\Delta_{j} v\right\|_{L^{2}} \\
& \quad \leq C\|v\|_{L^{2}}\left(\left\|\omega^{1}\right\|_{B_{\infty, 1}^{0, \gamma}}+\left\|\omega^{2}\right\|_{B_{\infty, 1}^{0, \gamma}}\right)+2^{j \alpha}\|\theta\|_{B_{2, \infty}^{-\alpha}} .
\end{aligned}
$$

Integrating in time yields

$$
\begin{aligned}
\left\|\Delta_{j} v(t)\right\|_{L^{2}} \leq & e^{-2^{j \alpha} t}\left\|\Delta_{j} v(0)\right\|_{L^{2}}+\int_{0}^{t} e^{-2^{j \alpha}(t-s)} 2^{j \alpha}\|\theta(s)\|_{B_{2, \infty}^{-\alpha}} d s \\
& +C \int_{0}^{t} e^{-2^{j \alpha}(t-s)}\|v(s)\|_{L^{2}}\left(\left\|\omega^{1}(s)\right\|_{B_{\infty, 1}^{0, \gamma}}+\left\|\omega^{2}(s)\right\|_{B_{\infty, 1}^{0, \gamma}}\right) d s .
\end{aligned}
$$

Therefore,

$$
\begin{aligned}
\|v(t)\|_{B_{2, \infty}^{0}} \leq & \|v(0)\|_{B_{2, \infty}^{0}}+\sup _{0 \leq s \leq t}\|\theta(s)\|_{B_{2, \infty}^{-\alpha}} \\
& +C \int_{0}^{t}\|v(s)\|_{L^{2}}\left(\left\|\omega^{1}(s)\right\|_{B_{\infty}^{0, \gamma},}+\left\|\omega^{2}(s)\right\|_{B_{\infty}^{0, \gamma}, 1}\right) d s .
\end{aligned}
$$

This completes the proof.

At the end, we give the main theorem of this section.

Theorem 5.3. Assume that $\left(\omega_{0}, \theta_{0}\right)$ satisfies the conditions stated in Theorem 1.1. Let $\sigma=0, \gamma \geq 0$ and $q>2$. Let $\left(\omega^{1}, \theta^{1}\right)$ and $\left(\omega^{2}, \theta^{2}\right)$ be two solutions of (1.1) satisfying for any $t>0$,

$$
\omega^{1}, \omega^{2} \in L_{t}^{1} L^{2} \cap L_{t}^{1} B_{\infty, 1}^{0, \gamma}, \quad \theta^{1}, \theta^{2} \in L_{t}^{1} L^{2} \cap L_{t}^{1} B_{\infty, 1}^{0, \gamma} .
$$

Then they must coincide.

Proof. Using the notations stated in the beginning of this section, we know that $v, \theta, u$ and $p$ satisfy (5.1) and (5.4). In our deduction, we will use the following two inequalities

$$
\|v\|_{L^{2}} \leq C\|v\|_{B_{2, \infty}^{0}} \log \left(1+\frac{\|v\|_{H^{1}}}{\|v\|_{B_{2, \infty}^{0}}}\right)
$$

and

$$
\|v\|_{H^{1}} \leq\left\|\omega^{1}\right\|_{L^{2}}+\left\|\omega^{2}\right\|_{L^{2}} .
$$

Combining the inequalities above and setting

$$
Y(t)=\|\theta(t)\|_{B_{2, \infty}^{-\alpha}}+\|v(t)\|_{B_{2, \infty}^{0}}
$$

we obtain

$$
Y(t) \leq 2 Y(0)+C \int_{0}^{t} D_{1}(s)\left[Y(s) \log \left(1+\frac{D_{2}(s)}{Y(s)}\right)+Y(s)\right] d s,
$$

where

$$
\begin{aligned}
& D_{1}(s)=\left\|\theta^{1}(s)\right\|_{B_{\infty, 1}^{1-\alpha, \gamma}}+\left\|\omega^{1}(s)\right\|_{B_{\infty, 1}^{0, \gamma}}+\left\|\omega^{2}(s)\right\|_{B_{\infty, 1}^{0, \gamma}} \\
& D_{2}(s)=\left\|\omega^{2}(s)\right\|_{L^{2}}+\left\|\omega^{2}(s)\right\|_{L^{2}} .
\end{aligned}
$$


Using the same idea in the proof of the integrable of $\|\theta\|_{B_{\infty, 1}^{0, \gamma} \text {, we can }}$ prove the that $\left\|\theta^{1}(s)\right\|_{B_{\infty, 1}^{1-\alpha, \gamma}}$ is integrable. Hence, we know that $D_{1}$ and $D_{2}$ are integrable. By Osgood's inequality we get $Y(t)=0$. This completes the proof.

\section{Appendix: Technical Lemmas}

Here we give some useful estimates in Besov framework.

Lemma 6.1. Let $u$ be a smooth divergence-free vector field of $\mathbb{R}^{d}$ and $f$ be a smooth scalar function. Then

(1) for every $\alpha \in(\sigma+\epsilon, 1)$ and $p \in[2, \infty]$

$$
\begin{aligned}
& \sup _{q \geq-1} 2^{q(\alpha-1-\epsilon-\sigma)}\left\|\left[\Delta_{q}, u \cdot \nabla\right] f\right\|_{L^{p}} \\
& \quad \lesssim\left(\left\|\Lambda^{1-\sigma-\epsilon} u\right\|_{B_{p, \infty}^{\alpha-1}}+\|u\|_{L^{2}}\right)\|f\|_{B_{\infty, \infty}^{0}}
\end{aligned}
$$

(2) for a special $u=\nabla^{\perp} \Delta^{-1} \Lambda^{\sigma}(\log (I d-\Delta))^{\gamma} \omega$

$$
\begin{aligned}
& \sup _{q \geq-1} 2^{q(\alpha-1-\epsilon-\sigma)}\left\|\left[\Delta_{q}, u \cdot \nabla\right] f\right\|_{L^{p}} \\
& \quad \lesssim\left(\|G\|_{B_{p, \infty}^{\alpha-1}}+\|G\|_{L^{2}}+\left\|\theta_{0}\right\|_{L^{p} \cap L^{2}}\right)\|f\|_{B_{\infty, \infty}^{0}} .
\end{aligned}
$$

Proof. (1) From Bony's decomposition we have

$$
\begin{aligned}
{\left[\Delta_{q}, u \cdot \nabla\right] f=} & \sum_{|j-q| \leq 4}\left[\Delta_{q}, S_{q-1} u \cdot \nabla\right] \Delta_{j} f+\sum_{|j-q| \leq 4}\left[\Delta_{q}, \Delta_{j} u \cdot \nabla\right] S_{j-1} f \\
& +\sum_{\substack{j \geq q-3,1 \leq i \leq n\\
}}\left[\Delta_{q} \partial_{i}, \Delta_{j} u^{i}\right] \widetilde{\Delta}_{j} f \\
= & I_{q}+I I_{q}+I I I_{q} .
\end{aligned}
$$

Estimation of $I_{q}$. Since $\Delta_{q}:=h_{q}(\cdot) *=2^{q d} h\left(2^{q} \cdot\right) *$ with $h \in \mathcal{S}\left(\mathbb{R}^{d}\right)$, then from Lemma 3.2 we get for every $\alpha<1$

$$
\begin{aligned}
\left\|I_{q}\right\|_{L^{p}} \lesssim & \sum_{|j-q| \leq 4}\left\||x|^{1-\sigma-\epsilon} \phi_{q}\right\|_{L^{1}}\left\|\Lambda^{1-\sigma-\epsilon} S_{j-1} u\right\|_{L^{p}} 2^{j}\left\|\Delta_{j} f\right\|_{L^{\infty}} \\
\lesssim & \|f\|_{B_{\infty}^{0}, \infty}\left\||x|^{1-\sigma-\epsilon} \phi\right\|_{L^{1}} \sum_{|j-q| \leq 4} 2^{(j-q)(1-\sigma-\epsilon)} 2^{j(1+\epsilon+\sigma-\alpha)} \\
& \times \sum_{k \leq j-2} 2^{(j-k)(\alpha-1)} 2^{k(\alpha-1)}\left\|\Delta_{k} \Lambda^{1-\sigma-\epsilon} u\right\|_{L^{p}} \\
\lesssim & 2^{q(1+\epsilon+\sigma-\alpha)}\left\|\Lambda^{1-\sigma-\epsilon} u\right\|_{B_{p, \infty}-1}^{\alpha-1}\|f\|_{B_{\infty, \infty}^{0}},
\end{aligned}
$$

thus we have

$$
\sup _{q \geq-1} 2^{q(\alpha-1-\epsilon-\sigma)}\left\|I_{q}\right\|_{L^{p}} \lesssim\left\|\Lambda^{1-\sigma-\epsilon} u\right\|_{B_{p, \infty}^{\alpha-1}}\|f\|_{B_{\infty, \infty}^{0}} .
$$


Estimation of $I I_{q}$. Similar to the estimation of $I_{q}$, we can obtain

$$
\begin{aligned}
\left\|I I_{q}\right\|_{L^{p}} & \lesssim \sum_{|j-q| \leq 4}\left\||x|^{1-\sigma-\epsilon} \phi_{q}\right\|_{L^{1}}\left\|\Lambda^{1-\sigma-\epsilon} \Delta_{j} u\right\|_{L^{p}}\left\|S_{j-1} \nabla f\right\|_{L^{\infty}} \\
& \lesssim\left\|\Lambda^{1-\sigma-\epsilon} u\right\|_{B_{p, \infty}^{\alpha-1}} \sum_{|j-q| \leq 4}\left\||x|^{1-\sigma-\epsilon} \phi_{q}\right\|_{L^{1}} 2^{j(1-\alpha)}\left\|S_{j-1} \nabla f\right\|_{L^{\infty}} \\
& \lesssim\left\|\Lambda^{1-\sigma-\epsilon} u\right\|_{B_{p, \infty}^{\alpha-1}} \sum_{|j-q| \leq 4} 2^{-q(1-\sigma-\epsilon)} 2^{j(1-\alpha)} \sum_{k \leq j-2} 2^{k}\left\|\Delta_{k} f\right\|_{L^{\infty}} \\
& \lesssim 2^{q(1-\alpha+\sigma+\epsilon)}\left\|\Lambda^{1-\sigma-\epsilon} u\right\|_{B_{p, \infty}^{\alpha-1}}\|f\|_{B_{\infty}^{0}, \infty},
\end{aligned}
$$

thus

$$
\sup _{q \geq-1} 2^{q(\alpha-1-\sigma-\epsilon)}\left\|I I_{q}\right\|_{L^{p}} \lesssim\left\|\Lambda^{1-\sigma-\epsilon} u\right\|_{B_{p, \infty}^{\alpha-1}\|f\|_{B_{\infty, \infty}^{0}}}
$$

Estimation of $I I I_{q}$. We further write

$$
\begin{aligned}
I I I_{q} & =\sum_{j \geq q-3, j \in \mathbb{N}, 1 \leq i \leq d}\left[\Delta_{q} \partial_{i}, \Delta_{j} u^{i}\right] \widetilde{\Delta}_{j} f+\sum_{1 \leq i \leq d}\left[\Delta_{q} \partial_{i}, \Delta_{-1} u^{i}\right] \widetilde{\Delta}_{-1} f \\
& =I I I_{q}^{1}+I I I_{q}^{2} .
\end{aligned}
$$

For the first term, we get for every $\alpha>0$

$$
\begin{aligned}
& \left\|I I I_{q}^{1}\right\|_{L^{p}} \\
& \leq \sum_{j \geq q-3, j \in \mathbb{N}, 1 \leq i \leq d}\left\|\partial_{i} \Delta_{q}\left(\Delta_{j} u^{i}\right) \widetilde{\Delta}_{j} f\right\|_{L^{p}} \\
& +\sum_{j \geq q-3, j \in \mathbb{N}, 1 \leq i \leq d}\left\|\Delta_{j} u^{i} \partial_{i} \Delta_{q} \widetilde{\Delta}_{j} f\right\|_{L^{p}} \\
& \lesssim 2^{q(1+\epsilon+\sigma-\alpha)} \sum_{j \geq q-3, j \in \mathbb{N}} 2^{(q-j)(\alpha-\epsilon-\sigma)} 2^{j(\alpha-1)}\left\|\Delta_{j} \Lambda^{1-\sigma-\epsilon} u\right\|_{L^{p}}\left\|\widetilde{\Delta}_{j} f\right\|_{L^{\infty}} \\
& \lesssim 2^{q(1-\alpha+\sigma+\epsilon)}\left\|\Lambda^{1-\sigma-\epsilon} u\right\|_{B_{p, \infty}^{\alpha-1}}\|f\|_{B_{\infty, \infty}^{0}}
\end{aligned}
$$

thus

$$
\sup _{q \geq-1} 2^{q(\alpha-1-\epsilon-\sigma)}\left\|I I I_{q}^{1}\right\|_{L^{p}} \lesssim\left\|\Lambda^{1-\sigma-\epsilon} u\right\|_{B_{p, \infty}^{\alpha-1}}\|f\|_{B_{\infty, \infty}^{0}} .
$$

For the second term, due to $I I I_{q}^{2}=0$ for every $q \geq 3$, we get for $p \geq 2$

$$
\begin{aligned}
& \sup _{q \geq-1} 2^{q(\alpha-1-\epsilon-\sigma)}\left\|I I I_{q}^{2}\right\|_{L^{p}} \\
& \quad=\sup _{q \geq-1} 2^{q(\alpha-1-\epsilon-\sigma)}\left\|\left[\Delta_{q} \partial, \Delta_{-1} u\right] \widetilde{\Delta}_{-1} f\right\|_{L^{p}} \\
& \quad \lesssim\|u\|_{L^{2}}\|f\|_{B_{\infty, \infty}^{0}} .
\end{aligned}
$$

Hence, the proof is complete. 
(2) Using Bony's decomposition, we obtain same formula as in the proof of (1). Estimation of $I_{q}$. Similar to the proof of (1), we have

$$
\begin{aligned}
\left\|I_{q}\right\|_{L^{p}} \lesssim & \|f\|_{B_{\infty, \infty}^{0}} \sum_{|j-q| \leq 4} 2^{(j-q)(1-\sigma-\epsilon)} 2^{j(1+\epsilon+\sigma-\alpha)} \\
& \times \sum_{k \leq j-2} 2^{(j-k)(\alpha-1)} 2^{k(\alpha-1)}\left(\left\|\Delta_{k} G\right\|_{L^{p}}+\left\|\Delta_{k} \mathcal{R}_{\alpha} \theta\right\|_{L^{p}}\right) \\
\lesssim & 2^{q(1+\epsilon+\sigma-\alpha)}\|G\|_{B_{p, \infty}^{\alpha-1}}\|f\|_{B_{\infty, \infty}^{0}} \\
& +\|f\|_{B_{\infty}^{0}, \infty} \sum_{|j-q| \leq 4} 2^{(j-q)(1-\sigma-\epsilon)} 2^{j(1+\epsilon+\sigma-\alpha)} \sum_{k \leq j-2} 2^{(j-k)(\alpha-1)}\|\theta\|_{L^{p}} \\
\lesssim & 2^{q(1+\epsilon+\sigma-\alpha)}\|f\|_{B_{\infty, \infty}^{0}}\left(\|G\|_{B_{p, \infty}^{\alpha-1}}+\|\theta\|_{L^{p}}\right),
\end{aligned}
$$

thus

$$
\sup _{q \geq-1} 2^{q(\alpha-1-\epsilon-\sigma)}\left\|I_{q}\right\|_{L^{p}} \lesssim\left(\|G\|_{B_{p, \infty}^{\alpha-1}}+\|\theta\|_{L^{p}}\right)\|f\|_{B_{\infty, \infty}^{0}} .
$$

For $I I_{q}$, we have

$$
\begin{aligned}
\left\|I I_{q}\right\|_{L^{p}} & \lesssim \sum_{|j-q| \leq 4}\left\||x|^{1-\sigma-\epsilon} \phi_{q}\right\|_{L^{1}}\left\|\Delta_{j} \Lambda^{1-\sigma-\epsilon} u\right\|_{L^{p}}\left\|S_{j-1} \nabla f\right\|_{L^{\infty}} \\
& \lesssim \sum_{|j-q| \leq 4} 2^{-q(1-\sigma-\epsilon)}\left\|\Delta_{j} \Lambda^{1-\sigma-\epsilon} u\right\|_{L^{p}}\left\|S_{j-1} \nabla f\right\|_{L^{\infty}} \\
& \lesssim \sum_{|j-q| \leq 4} 2^{-q(1-\sigma-\epsilon)}\left(\left\|\Delta_{j} G\right\|_{L^{p}}+\left\|\Delta_{j} \mathcal{R}_{\alpha} \theta\right\|_{L^{p}}\right) \sum_{k \leq j-2} 2^{k}\left\|\Delta_{k} f\right\|_{L^{\infty}} \\
& \lesssim \sum_{|j-q| \leq 4} 2^{-q(1-\sigma-\epsilon)} 2^{j(1-\alpha)} \sum_{k \leq j-2} 2^{k}\left\|\Delta_{j} f\right\|_{L^{\infty}}\left(\|G\|_{B_{p, \infty}^{\alpha-1}}+\|\theta\|_{L^{p}}\right) \\
& \lesssim 2^{q(1-\alpha+\epsilon+\sigma)}\left(\|G\|_{B_{p, \infty}^{\alpha-1}}+\|\theta\|_{L^{p}}\right)\|f\|_{B_{\infty, \infty}^{0}},
\end{aligned}
$$

thus

$$
\sup _{q \geq-1} 2^{q(\alpha-1-\epsilon-\sigma)}\left\|I_{q}\right\|_{L^{p}} \lesssim\left(\|G\|_{B_{p, \infty}^{\alpha-1}}+\|\theta\|_{L^{p}}\right)\|f\|_{B_{\infty, \infty}^{0}}
$$

For the term $I I I_{q}^{1}$. We can calculus as follows.

$$
\begin{aligned}
& \left\|I I I_{q}^{1}\right\|_{L^{p}} \\
& \lesssim 2^{q(1-\alpha+\sigma+\epsilon)} \sum_{j \geq q-3, j \in \mathbb{N}} 2^{(q-j)(\alpha-\sigma-\epsilon)} 2^{j(\alpha-1)}\left\|\Delta_{j} \Lambda^{1-\sigma-\epsilon} u\right\|_{L^{p}}\left\|\widetilde{\Delta}_{j} f\right\|_{L^{\infty}} \\
& \lesssim 2^{q(1-\alpha+\sigma+\epsilon)} \sum_{j \geq q-3, j \in \mathbb{N}} 2^{(q-j)(\alpha-\epsilon-\sigma)} 2^{j(\alpha-1)}\left\|\Delta_{j} G\right\|_{L^{p}}\left\|\widetilde{\Delta}_{j} f\right\|_{L^{\infty}} \\
& \quad+2^{q(1-\alpha+\sigma+\epsilon)} \sum_{j \geq q-3, j \in \mathbb{N}} 2^{(q-j)(\alpha-\sigma-\epsilon)} 2^{j(\alpha-1)}\left\|\Delta_{j} \mathcal{R}_{\alpha} \theta\right\|_{L^{p}}\left\|\widetilde{\Delta}_{j} f\right\|_{L^{\infty}} \\
& \lesssim 2^{q(1-\alpha+\sigma+\epsilon)}\left(\|G\|_{B_{p, \infty}^{\alpha-1}}+\|\theta\|_{L^{p}}\right)\|f\|_{B_{\infty, \infty}^{0}} \cdot
\end{aligned}
$$



we have

For the term $I I I_{q}^{2}$. For every $q \geq 3$ we know that $I I I_{q}^{2}=0$. So for $p \geq 2$,

$$
\begin{aligned}
\sup _{q \geq-1} & 2^{q(\alpha-1-\epsilon-\sigma)}\left\|I I I_{q}^{2}\right\|_{L^{p}} \\
& =\sup _{q \geq-1} 2^{q(\alpha-1-\sigma-\epsilon)}\left\|\left[\Delta_{q} \partial, \Delta_{-1} u\right] \widetilde{\Delta}_{-1} f\right\|_{L^{p}} \\
& \lesssim\left\|\Delta_{-1} u\right\|_{L^{p}}\|f\|_{B_{\infty, \infty}^{0}} \\
& \lesssim\left\|\Delta_{-1} \Lambda^{\sigma+\epsilon-1} u\right\|_{L^{p}}\|f\|_{B_{\infty, \infty}^{0}} \\
& \lesssim\left(\left\|\Delta_{-1} G\right\|_{L^{p}}+\left\|\Delta_{-1} \mathcal{R}_{\alpha} \theta\right\|_{L^{p}}\right)\|f\|_{B_{\infty, \infty}^{0}} \\
& \lesssim\left(\|G\|_{L^{2}}+\left\|\theta_{0}\right\|_{L^{2}}\right)\|f\|_{B_{\infty, \infty}^{0}} .
\end{aligned}
$$

From all the above statements, we can obtain our results.

\section{Acknowledgements}

J. Jia would like to thank China Scholarship Council that has provided a scholarship for his research work in the United States. The first and second authors were supported by the National Science Foundation of China under the grants no. 11131006, 41390450, 91330204 and supported partially by the National Basic Research Program of China under the grant no. 2013CB329404. The third author was sponsored by the National Science Foundation of China under the grants no. 11201136, and China Postdoctoral Science Foundation Funded Project under the contact No. 2012M520080.

\section{References}

[1] Chae, D., Wu, J.: The 2D Boussinesq equations with logarithmically supercritical velocities. Adv. Math. 230, 1618-1645 (2012)

[2] Pedloshy, J.: Geophysical Fluid Dynamics. Springer, New York (1987)

[3] Chae, D.: Global regularity for the 2D Boussinesq equations with partial viscosity terms. Adv. Math. 203, 497-513 (2006)

[4] Hmidi, T., Keraani, S., Rousset, F.: Global well-posedness for a BoussinesqNavier-Stokes system with critical dissipation. J. Differ. Equ. 249, 2147$2174(2010)$

[5] Hmidi, T, Keraani, S., Rousset, F.: Global well-posedness for Euler-Boussinesq system with critical dissipation. Commun. Partial Differ. Equ. 36, 420$445(2011)$

[6] Miao, C., Xue, L.: On the global well-posedness of a class of Boussinesq-NavierStokes systems. Nonlinear Differ. Equ. Appl. NoDEA. 18, 707-735 (2011) 
[7] Wang, C., Zhang, Z.: Global well-posedness for the 2D Boussinesq system with the temperature-dependent viscosity and thermal diffusivity. Adv. Math. 228, 43-62 (2011)

[8] Hmidi, T., Rousset, F.: Global well-posedness for the Euler-Boussinesq system with axisymmetric data. J. Funct. Anal. 260, 745-796 (2011)

[9] Lai, M., Pan, R., Zhao, K.: Initial boundary value problem for two-dimensional viscous Boussinesq equations. Arch. Ration. Mech. Anal. 199, 739-760 (2011)

[10] Wu, G., Xue, L.: Global well-posedness for the 2D inviscid Bénard system with fractional diffusivity and Yudovich's type data. J. Differ. Equ. 253, 100$125(2012)$

[11] Bahouri, H., Chemin, J.-Y., Danchin, R.: Fourier Analysis and Nonlinear Partial Differential Equations. Grundlehren Math. Wiss. vol. 343, Springer (2011)

[12] Chemin, J.-Y.: Perfect Incompressible Fluids. Clarendon Press, Oxford (1998)

[13] Hmidi, T., Keraani, S., Rousset, F.: Global well-posedness for a BoussinesqNavier-Stokes system with critical dissipation. J. Differ. Equ. 249, 2147$2174(2010)$

[14] Córdoba, A., Córdoba, D.: A maximum principle applied to quasi-geostrophic equations. Commun. Math. Phys. 249, 511-528 (2004)

[15] Hmidi, T., Keraani, S.: On the global well-posedness of the two-dimensional Boussinesq system with a zero viscosity. Indiana Univ. Math. J. 58(4), 15911618 (2007)

[16] Miao, C., Wu, G.: Global well-posedness of the critical Burgers equation in critical Besov spaces. J. Differ. Equ. 247, 1673-1693 (2009)

[17] Ju, N.: The maximum principle and the global attractor for the dissipative 2D quasi-geostrophic equations. Commun. Math. Phys. 255, 161-181 (2005)

[18] Chen, Q., Miao, C., Zhang, Z.: A new Bernstein's inequality and the 2D dissipative quasi-geostrophic equation. Commun. Math. Phys. 271, 821-838 (2007)

Junxiong Jia, Jigen Peng and Kexue Li

Department of Mathematics

Xi'an Jiaotong University

Xi'an 710049

China

e-mail: jjx425@gmail.com

Junxiong Jia, Jigen Peng and Kexue Li

Beijing Center for Mathematics and Information Interdisciplinary

Sciences (BCMIIS)

Beijing

China 
Vol. 22 (2015)

J. Peng

e-mail: jgpeng@mail.xjtu.edu.cn

K. $\mathrm{Li}$

e-mail: kexueli@gmail.com

Received: 9 October 2013.

Accepted: 23 December 2014. 